\title{
Elliptic gaussian random processes
}

Albert Benassi, Stéphane Jaffard and Daniel Roux

\begin{abstract}
We study the Gaussian random fields indexed by $\mathbb{R}^{d}$ whose covariance is defined in all generality as the parametrix of an elliptic pseudo-differential operator with minimal regularity asumption on the symbol. We construct new wavelet bases adapted to these operators; the decomposition of the field on this corresponding basis yields its iterated logarithm law and its uniform modulus of continuity. We also characterize the local scalings of the field in term of the properties of the principal symbol of the pseudodifferential operator. Similar results are obtained for the Multi-Fractional Brownian Motion.
\end{abstract}

Résumé. Nous étudions les processus aléatoires gaussiens $X$ indexés par $\mathbb{R}^{d}$ tels qu'il existe un opérateur pseudo-différentiel $A$ d'ordre donné admettant pour parametrix la covariance de $X$.

Nous construisons une base d'ondelettes adaptée à l'opérateur $A$. La décomposition du processus $X$ sur cette base conduit à la loi du module de continuité uniforme et à la loi du logarithme itéré. Nous caractérisons aussi les propriétés d'échelle locales du processus au moyen de la partie principale du symbole. Nous étendons ces résultats au Mouvement Brownien Multi-Fractionnaire qui est associé à un opérateur d'ordre variable. 


\section{Introduction and statement of results.}

\subsection{Introduction.}

Let $X(x)$ be a (centered real valued) Gaussian Random Process defined on $\mathbb{R}^{d}$, of covariance $R(x, y)=\mathbb{E}(X(x) X(y))$. Two isomorphic Hilbert spaces are associated with $X$ : the space $\mathcal{H}$ defined by the closure of the random variables $Z=\sum \alpha_{i} X\left(t_{i}\right)$ for the scalar product $(Z \mid T)=\mathbb{E}(Z T)$ and the Reproducing Kernel Hilbert Space (R.K.H.S.) $H$ composed of the functions which can be written

$$
f_{Z}(t)=\mathbb{E}(X(t) Z)
$$

with $Z \in \mathcal{H}$; the scalar product in $H$ is

$$
\left(f_{Z}, f_{Y}\right)_{H}=\mathbb{E}(Z Y) \text {. }
$$

By Riesz's representation theorem, we can define a self-adjoint positive operator $A: H \rightarrow H^{\prime}$, the dual of $H$ by

$$
(f, g)_{H}=\langle A(f) \mid g\rangle_{\left(H^{\prime}, H\right)},
$$

where $\langle\cdot \mid \cdot\rangle_{\left(H^{\prime}, H\right)}$ means the $\left(H^{\prime}, H\right)$ duality.

Of particular significance is the case where the norm in $H$ is equivalent with the norm of one of the Sobolev spaces $H^{s}$ or of the homogeneous spaces $\dot{H}^{s}$ (in this last case $H$ is defined by additional conditions, for instance by vanishing conditions at the origin). We will call Elliptic Gaussian Random Processes the processes such that

$$
C_{1}\|f\|_{\dot{H}^{s}}^{2} \leq(A(f) \mid f)_{L^{2}} \leq C_{2}\|f\|_{H^{s}}^{2},
$$

which is an ellipticity asumption on the operator $A$ (we borrow this terminology from Guyon [17] where it covers a similar idea). These norm estimates imply that the operator is everywhere of order $2 s$. We will show later that the techniques we introduce allow also to study the Multifractional Brownian Motion, a case where the order of the operator is a function of $x$.

We specify the setting by requiring $A$ to be a pseudodifferential operator, and we will make some limited regularity asumptions on its symbol $\sigma(x, \xi)$. Since theoretically all the information on $X$ is contained in the operator $A$, we want to investigate in details the correspondence 
between the properties of $X$ and $A$. Some points are classical; for instance $X$ has the Markov field property if and only if $A$ is differential; $X$ has stationary increments if and only if the symbol $\sigma(x, \xi)$ does not depend on $x$ (the norm in $H$ is then shift-invariant).

In this work, we will mainly study two properties of the process:

1) local self-similarity,

2) regularity of the sample paths, looking for exact constants in the laws of local and uniform moduli of continuity.

Let us recall that a process $X$ is said to be selfsimilar of order $\alpha$ at the origin if, for all $\rho>0$,

$$
\text { Law }\left\{\rho^{-\alpha} X(\rho x), x \in \mathbb{R}^{d}\right\}=\operatorname{Law}\left\{X(x), x \in \mathbb{R}^{d}\right\} .
$$

For instance, the Fractional Brownian Motion of order $\alpha$ is selfsimilar (of order $\alpha$ ) at the origin. Dobrushin in [13] gives a complete characterization of selfsimilar gaussian fields with stationary increments; and it follows from [13] that the exact scaling law (3) can hold only for very specific processes. The renormalisation operators $R_{x_{0}, r}^{\alpha}$ are defined by

$$
R_{x_{0}, \rho}^{\alpha} X(x)=\frac{1}{\rho^{\alpha}}\left(X\left(x_{0}+\rho x\right)-X\left(x_{0}\right)\right)
$$

and, by definition, a process $X$ is locally asymptotically self-similar (L.A.S.S.) of order $\alpha \in(0,1)$ at $x_{0}$ if $R_{x_{0}, \rho}^{\alpha} X$ has a non trivial limit in law when $\rho \rightarrow \infty$.

The case $\alpha=1$ requires a different renormalisation formula, see (39), and the corresponding processes will be called locally asymptotically critical processes (L.A.C.).

Regularity properties for Gaussian processes have been considered in full generality in [16]. In the general case the uniform and local moduli of continuity are known only up to a multiplicative constant. For a large class of stationary increments processes Kono in [26] and Marcus in [29] obtain the exact constants in laws of the moduli, but these cases do not include in the elliptic setting the critical order $s-d / 2 \in \mathbb{N}$. One of our purposes is to solve completely this problem (Theorem 1.3) in the general elliptic pseudodifferential setting.

One of the main ideas behind the results we will describe is that the local properties of an elliptic gaussian process are contained in the principal part of the symbol associated with the operator $A$. Let us illustrate this idea on a very simple example. 
Let $W$ be the one dimensional Brownian motion issued from 0 . The corresponding operator $A_{0}$ is the second derivative, whose symbol $\sigma_{0}(x, \xi)$ is $|\xi|^{2}$. The fact that $\sigma_{0}$ is an homogeneous function of $\xi$ independent of $x$ implies that $W$ is selfsimilar of degree $1 / 2$. The Orstein-Uhlenbeck process $V$ is the solution of the stochastic differential equation $d V=-q V d t+d W$. One easily checks that the R.K.H.S. of this process is exactly the Sobolev space $H^{1}$ so that the corresponding operator is $A=q^{2} \operatorname{Id}-\partial^{2} / \partial x^{2}$ of symbol $\sigma(x, \xi)=|\xi|^{2}+q^{2}$. Since the modification of the symbol bears on low order terms only, for every bounded open subset $U$ of $\mathbb{R}$, Law $\left(\left.V\right|_{U}\right) \equiv \operatorname{Law}\left(\left.W\right|_{U}\right)$ (the two processes restricted to $\mathrm{U}$ are locally undistinguishable on one realization), see [32]. This has two more consequences:

1) The Orstein-Uhlenbeck process $V$ will satisfy the following local scaling property at any point $t$

$$
\lim _{\rho \rightarrow 0^{+}} \operatorname{Law}\left\{\frac{V(t+\rho u)-V(t)}{\rho^{1 / 2}}, u \in \mathbb{R}\right\}=\operatorname{Law} W,
$$

and we observe that the symbol of the "asymptotic process" is the "principal part" of $\sigma$.

2) The uniform modulus of continuity and the iterated logarithm law (the local modulus of continuity) of $V$ and $W$ are the same.

\subsection{The Model.}

In this paper we consider triples $\left(A, H_{A}, X_{A}\right)$ constituted by

- An elliptic symetric positive pseudodifferential operator $A$ derived from a symbol $\sigma: \mathbb{R}^{d} \times \mathbb{R}^{d} \rightarrow \mathbb{R}$ by the usual formula

$$
(A f)(x)=\frac{1}{(2 \pi)^{d / 2}} \int e^{i x \xi} \sigma(x, \xi) \hat{f}(\xi) d \xi,
$$

where $\hat{f}$ is the Fourier transform of $f$. We will use the notation $A=$ $\mathrm{O} p(\sigma)$.

- A Hilbert space $H_{A}$ whose scalar product is given by the generalized Dirichlet form

$$
\mathcal{A}(f, g)=\int A(f)(x) \bar{g}(x) d x
$$


defined at least for $f, g \in \mathcal{D}$, with $\mathcal{D}=\mathcal{D}\left(\mathbb{R}^{d}\right)$.

- A gaussian centered process $X_{A}$ with $H_{A}$ as Reproducing Kernel Hilbert Space (R.K.H.S.), see [32]. The covariance function $r$ of $X_{A}$

$$
r(x, y)=\mathbb{E}\left(X_{A}(x) X_{A}(y)\right),
$$

is the kernel of $A^{-1}$ (defined on appropriate spaces) and a parametrix of the operator $A$.

Let us now define a class of symbols and state some precise asumptions for the symbols $\sigma$ we use.

Definition 1.1. A symbol $\sigma$ defined on $\mathbb{R}^{d} \times \mathbb{R}^{d} \backslash\{0\}$ belongs to $S_{n}^{m}$, $m \in \mathbb{R}, n \in \mathbb{N}$ if,

1) for any multi-index $s$ with length $|s|=s_{1}+\cdots+s_{d} \leq n$, exists $C_{s}$ such that

$$
\left|\partial_{\xi}^{s} \sigma(x, \xi)\right| \leq C_{s}(1+|\xi|)^{m-|s|}, \quad \text { for } \xi \neq 0,
$$

2) there exists $\varepsilon>\varepsilon^{\prime} \geq 0$ such that

$$
|\sigma(x, \xi)-\sigma(y, \xi)| \leq C(1+|\xi|)^{m+\varepsilon^{\prime}}|x-y|^{\varepsilon} .
$$

Note that these properties are not the minimal asumptions which imply continuity of the operator between Sobolev spaces (see [12] for such conditions). But they are the minimal regularity asumptions implying that the symbol behaves "locally as if it were constant in $x$ at high frequencies", a fact we will need to characterize the local scaling properties of the process $X$.

Hypothesis HA $(m, \gamma)$. Let $m \geq 0$ and $\gamma>0 . A=O p(\sigma)$ satisfies hypothesis $\mathrm{HA}(m, \gamma)$ if

- $\sigma \in S_{[\gamma]+d+1}^{m}$,

- there exists $c>0$ such that

$$
c|\xi|^{m} \leq \sigma(x, \xi) .
$$

- There exist $C_{1}$ and $C_{2}>0$ such that

$$
C_{1} \int|\xi|^{m}|\hat{f}(\xi)|^{2} d \xi \leq(A(f) \mid f)_{L^{2}} \leq C_{2} \int(1+|\xi|)^{m}|\hat{f}(\xi)|^{2} d \xi
$$


REmARKs. a) The last inequalities can be rewritten as

$$
C_{1}\|f\|_{\dot{H}^{m / 2}}^{2} \leq(A(f) \mid f)_{L^{2}} \leq C_{2}\|f\|_{H^{m / 2}}^{2} .
$$

b) The hypothesis HA $(m, \gamma)$ is related to the existence of a dual process for $X$ (see [31] or [23]).

Hypothesis HAS $(m, \gamma)$. We say $\operatorname{HAS}(m, \gamma)$ is satisfied when there exists $c>0$, such that

$$
c\left(1+|\xi|^{m}\right) \leq \sigma(x, \xi), \quad \text { if } \xi \neq 0
$$

and

$$
(A(f) \mid f)_{L^{2}} \sim\|f\|_{H^{m / 2}}^{2} .
$$

The Model under Hypothesis $\operatorname{HAS}(m, \gamma)$ When $m>d$. Let us suppose $m>d$ and $\operatorname{HAS}(m, \gamma)$ holds for the symbol $\sigma$. Let $H_{A}=$ $\operatorname{cl}_{\mathcal{A}}(\mathcal{D})$, the closure of $\mathcal{D}=\mathcal{D}\left(R^{d}\right)$ with respect to the inner product $\mathcal{A}$. Then $H^{m / 2} \equiv H_{A} \subset L^{2} \subset H_{A}^{\prime}$ and $A^{-1}: H_{A}^{\prime} \rightarrow H_{A}$ can be written using the kernel theorem as

$$
A^{-1} f(x)=\int r(x, y) f(y) d y,
$$

with $r$ a continuous kernel on $\left(R^{d}\right)^{2}$. As $r$ is symetric and of positive type we know (see [32]) that there exists a centered gaussian process $X_{A}$ with covariance function $r$. The triple $\left(A, H_{A}, X_{A}\right)$ satisfies the conditions we ask for our model.

The model under Hypothesis HA $(m, \gamma)$ When $m>d$. Let us now suppose only HA $(m, \gamma)$ holds. As the operator $A$ may be non-inversible, a definition of $H_{A}$ requires more care. In [9] Bourdaud gives a dilation invariant realization of the homogeneous Sobolev space $\dot{H}^{m / 2}$. Similar ideas will be used here. Let us start with the case that turns out to be the most important for us, for it leads to processes which are limits in law of local renormalisations.

Suppose that $m-d=2(l+\alpha)>0$ with $l$ an integer and $\alpha \in$ $(0,1)$. Let $\sigma(\xi)=|\xi|^{m} S^{2}(\xi /|\xi|), A=O p(\sigma)$, where the function $S$ is continuous on the unit sphere of $R^{d}$ and takes only positive values. In this case we set $H=\operatorname{cl}_{\mathcal{A}}\left(\mathcal{D}_{0}\right)$ with

$$
\mathcal{D}_{0}=\left\{\psi \in \mathcal{D}: D^{\beta} \psi(0)=0, \text { if }|\beta|<\frac{m-d}{2}\right\} .
$$


Let $W$ be a gaussian white noise on $R^{d}$. The gaussian process $X_{x}$ is defined by

$$
X_{x}=\int \exp _{l}(i x \xi) \hat{\varphi}(\xi) \frac{d \overline{\hat{W}}(\xi)}{\sqrt{\sigma(\xi)}}
$$

where $\exp _{l}(y)=e^{y}-\sum_{0 \leq k \leq l} y^{k} / k$ !. For every $\varphi \in L^{2}$ let

$$
f_{\varphi}(x)=\int \exp _{l}(i x \xi) \hat{\varphi}(\xi) \frac{d \xi}{\sqrt{\sigma(\xi)}} .
$$

The following result shows that $(A, H, X)$ fullfills our conditions.

Lemma 1.1. The symbol $\sigma$ satisfies $\mathrm{HA}(m, \gamma)$. The Hilbert space $H$ is the R.K.H.S. of the gaussian process $X$ and we have

$$
\begin{gathered}
H=\left\{f_{\varphi}: \varphi \in L^{2}\right\}, \\
\mathcal{A}\left(f_{\varphi_{1}}, f_{\varphi_{2}}\right)=\left(\varphi_{1} \mid \varphi_{2}\right)_{L^{2}} .
\end{gathered}
$$

As this lemma can be deduced from results of [13] we only sketch the proof. It is easy to check that $\left\{f_{\varphi}, \varphi \in L^{2}\right\}$ is a Hilbert space with $\mathcal{A}$ as inner product and that (10) holds. We can also notice that for $\psi \in \mathcal{D}_{0}$

$$
\mathcal{A}\left(f_{\varphi}, \psi\right)=\left(\sqrt{\sigma} \hat{f}_{\varphi} \mid \sqrt{\sigma} \hat{\psi}\right)_{L^{2}}=(\hat{\varphi} \mid \sqrt{\sigma} \hat{\psi})_{L^{2}}
$$

Therefore if $\mathcal{A}\left(f_{\varphi}, \psi\right)=0$, for all $\psi \in \mathcal{D}_{0}$, we get $\hat{\varphi}=0$, and then $f_{\varphi}=0$. This shows that $\mathcal{D}_{0}$ is dense in $H$ and thus (9) holds. It remains to prove that $H$ is the R.K.H.S. of $X$, i.e. for all $x$ we have

$$
f_{\varphi}(x)=\mathcal{A}\left(K_{x}, f_{\varphi}\right)
$$

where $K_{x}(y)=E\left(X_{x} X_{y}\right)$ is the covariance function of the process $X$. Since $K_{x}(y)=f_{k_{x}}$, where

$$
\hat{k}_{x}(\xi)=\frac{\exp _{l}(-i x \xi)}{|\xi|^{m / 2} S(\xi /|\xi|)},
$$

thus

$$
\mathcal{A}\left(K_{x}, f_{\varphi}\right)=\left(\hat{\varphi} \mid \frac{\exp _{l}(-i x \xi)}{|\xi|^{m / 2} S(\xi /|\xi|)}\right)_{L^{2}}=f_{\varphi}(x)
$$


Remarks. When $S(\xi) \equiv 1$ and $l=0, X$ is the $d$-dimensional Fractional Brownian motion of order $\alpha$.

If $\alpha=1$, the integral (8) does not define a process any longer (to study this case, we would have to split the integral). This is coherent with the facts that in this case $\dot{H}^{l+1+d / 2}$ has no dilation invariant realization (see [9]) and that a different normalization is required for Elliptic Gaussian Processes of critical order $2 l+d(l \in \mathbb{N})$ to have a local asymptotic scaling law.

Finally one should notice that other spaces $H_{A}$ can be associated with a single operator $A=O p(\sigma)$. But the associated processes are locally the same. For example if Hypothesis HAS $(m, \gamma)$ holds, define $H_{A}$ (respectively $H_{A, 0}$ ) equal to $\operatorname{cl}_{\mathcal{A}}(\mathcal{D})$ (respectively $\mathrm{cl}_{\mathcal{A}}\left(\mathcal{D}_{0}\right)$ ) and denote by $X_{A}, X_{A, 0}$ the associated processes. For any open bounded subset $U \subset R^{d} \backslash\{0\}$ it is easy to see (think of the brownian motion and bridge) that the laws of the restricted processes are equivalent, that is

$$
\operatorname{Law}\left(\left.X_{A, 0}\right|_{U}\right) \equiv \operatorname{Law}\left(\left.X_{A}\right|_{U}\right)
$$

Convention. From now on we suppose the triple $\left(A, H_{A}, X_{A}\right)$ is given and satisfies the conditions of our model and, unless otherwise specified, Hypothesis HA $(m, \gamma)$.

\subsection{Outline of the method.}

The method we will use in order to obtain the modulus of continuity and the local scaling laws of the elliptic processes is the following.

a) For an operator $A$ satisfying $\operatorname{HAS}(m, \gamma)$ we will construct in Section 2 an orthonormal wavelet basis $\Phi_{\lambda}$ of $H_{A}$ indexed by the dyadic cubes, and such that each $\Phi_{\lambda}$ is localized near the corresponding dyadic cube (precise localization estimates are stated in Theorem 1.1 of Section 1.4). Using the canonical isomorphism between $\mathcal{H}$ and $H_{A}$ we get

$$
X_{A}(x)=\sum_{\lambda} \xi_{\lambda} \Phi_{\lambda}
$$

where the $\xi_{\lambda}$ are independant normalized centered Gaussian; In Section 4 , the local properties of the process $X_{A}$ will be deduced from this decomposition. 
b) In the general case $\mathrm{HA}(m, \gamma)$ we will perform a modification of the symbol at low frequencies in order to obtain a new process for which the stronger asumption $\operatorname{HAS}(m, \gamma)$ holds, and such that the two processes have the same local properties. This will be true because low frequency modifications do not alter such properties as local regularity or asymptotic scaling. Let us state the modification and prove this result. Let $g$ be a nonnegative function in $\mathcal{D}\left(\mathbb{R}^{n}\right)$ such that $\operatorname{supp}(g) \subset$ $B(0,2)$, and

$$
g(\xi)=1, \quad \text { if }|\xi| \leq 1
$$

Let $G$ be the operator of convolution with $\hat{g}$ and set

$$
A_{g}=(\mathrm{Id}-G) A(\mathrm{Id}-G)+G .
$$

Clearly, if $A$ is selfadjoint positive, so is $A_{g} \cdot X_{A}, X_{A_{g}}$ will denote the associated gaussian elliptic processes.

Proposition 1.1. The operator $A_{g}$ satisfies $\operatorname{HAS}(m, \gamma)$ and for any bounded open subset $U$ of $\mathbb{R}^{d}$ such that $0 \notin U$

$$
\operatorname{Law}\left(\left.X_{A}\right|_{U}\right) \equiv \operatorname{Law}\left(\left.X_{A_{g}}\right|_{U}\right)
$$

Proof of Proposition 1.1. The symbol $\sigma_{g}$ of $A_{g}$ is given by

$$
\sigma_{g}(x, \xi)=g(\xi)+(1-g(\xi))^{2} \sigma(x, \xi)+r(x, \xi),
$$

with $r(x, \xi)$ a regularizing kernel. It is easy to check that $\sigma_{g}$ fulfills the conditions of HA $(m, \gamma)$,

$$
C_{1} \int|\xi|^{2 s}|\hat{f}(\xi)|^{2} d \xi \leq(A(f) \mid f)_{L^{2}} \leq C_{2} \int\left(1+|\xi|^{2 s}\right)|\hat{f}(\xi)|^{2} d \xi
$$

The conditions for $\operatorname{HAS}(m, \gamma)$ are satisfied because

$$
\begin{aligned}
\left(A_{g}(f) \mid f\right)_{L^{2}} & \sim\|(\operatorname{Id}-G) f\|_{\dot{H}^{s}}^{2}+(G(f) \mid f)_{L^{2}} \\
& \sim \int(1-g(\xi))|\xi|^{2 s}|\hat{f}(\xi)|^{2} d \xi+\int g(\xi)|\hat{f}(\xi)|^{2} d \xi \\
& \sim \int(1+|\xi|)^{2 s}|\hat{f}(\xi)|^{2} d \xi
\end{aligned}
$$


Using (11) we can assume that the processes are starting from 0, i.e. the related R.K.H.S. are the closure of $\mathcal{D}_{0}$ for $\mathcal{A}$ and $\mathcal{A}_{g}$. The local equivalence result follows from [32, Theorem 8.6] if we check that

i) $C_{U}(x, y):=\left.A^{-1}\right|_{U \times U}(x, y)-\left.A_{g}^{-1}\right|_{U \times U}(x, y) \in H_{A_{g}}^{\otimes 2}(U \times U)$,

ii) -1 is not an eigenvalue of $C_{U}: H_{A_{g}} \rightarrow H_{A_{g}}$.

Let us consider the operator $B$

$$
B:=A-A_{g}=G A G-A G-G A+G .
$$

As the function $g$ belongs to $\mathcal{D}\left(\mathbb{R}^{d}\right)$ we know that $B$ is a regularizing operator;

$$
A^{-1}-A_{g}^{-1}=A_{g}^{-1}\left(\left(I+B A_{g}^{-1}\right)^{-1}-I\right)
$$

and

$$
\left(I+B A_{g}^{-1}\right)^{-1}=\sum_{n \geq 0}(-1)^{n}\left(B A_{g}^{-1}\right)^{n} .
$$

Now if we consider the restrictions to open bounded $U$ which are small enough, the last series converges and the operator $A^{-1}-A_{g}^{-1}$ is of Hilbert-Schmidt type with a spectral radius less than 1 , so that condition ii) is satisfied.

For the first condition, it is sufficient to show that

$$
(-\Delta)_{x}^{m / 4}(-\Delta)_{y}^{m / 4} C(x, y) \in L_{\mathrm{loc}}^{2}\left(\mathbb{R}^{d} \otimes \mathbb{R}^{d}\right)
$$

But, as before

$$
\begin{aligned}
(-\Delta)_{x}^{m / 4}(-\Delta)_{y}^{m / 4} C(x, y) & \\
& =\sum_{n \geq 1}(-1)^{n}(-\Delta)_{x}^{m / 4} A_{g}^{-1}\left(B A_{g}^{-1}\right)^{n}(-\Delta)_{x}^{m / 4}(x, y)
\end{aligned}
$$

which converges in $L^{2}(U \times U)$ for $U$ small enough, since $A_{g}^{-1}$ is an operator of order $-m$ and $B$ is regularizing.

Finally we obtain the equivalence of laws for every bounded open subset $U$ of $\mathbb{R}^{d} \backslash\{0\}$, by decomposing $U$ in a finite number of small enough open subsets. 


\subsection{Wavelets and pseudodifferential operators.}

We will construct a wavelet basis associated with an operator $A_{g}$ satisfying HAS $(m, \gamma)$. We obtain this basis by applying $A_{g}{ }^{-1 / 2}$ on the "Littlewood-Paley" orthonormal wavelet basis of $L^{2}$ defined by Lemarié and Meyer (see [30]). Let us recall some properties of this basis.

\subsubsection{The "Littlewood-Paley" wavelet basis.}

There exists $\phi$ and $\left.\psi^{(l)}, l \in L:=\{0,1\}^{d} \backslash\{(0, \ldots, 0)\}\right)$ such that $\hat{\phi}$ is $C^{\infty}$ and supported in the domain $|\xi| \leq 4 \pi / 3 ; \hat{\psi}^{(l)}$ are $C^{\infty}$ with support included in the domain $2 \pi / 3 \leq|\xi| \leq 8 \pi / 3$; the following translations and dilations of these functions

$$
\begin{gathered}
\phi_{k}(x)=\phi(x-k), \quad k \in \mathbb{Z}^{d}, \\
\psi_{j, k}^{(l)}(x)=2^{d j / 2} \psi^{(l)}\left(2^{j} x-k\right), \quad j \in \mathbb{N}, k \in \mathbb{Z}^{d}, l \in L,
\end{gathered}
$$

are an orthonormal basis of $L^{2}\left(\mathbb{R}^{d}\right)$ (notice that the family $\left\{\psi_{j, k}^{(l)}: j \in\right.$ $\left.\mathbb{Z}, k \in \mathbb{Z}^{d}, l \in L\right\}$ is also an orthonormal basis of $\left.L^{2}\left(\mathbb{R}^{d}\right)\right)$.

In order to simplify the notations, let $\psi_{0, k}^{(0, \ldots, 0)}:=\phi_{k}, k \in \mathbb{Z}^{d}$ and

$$
\psi_{\lambda}:=\psi_{j, k}^{(l)}, \lambda=(j, k, l) \in \mathbb{Z} \times \mathbb{Z}^{d} \times L \cup\{0\} \times \mathbb{Z}^{d} \times\{(0, \ldots, 0)\} .
$$

For a given $\lambda=(j, k, l)$, the integer $j$ will be often referred to as $j_{\lambda}$, and called the scale of $\lambda$. By abuse, $\lambda$ will often be identified with the dyadic point $\tilde{\lambda}=k 2^{-j}+l 2^{-j-1}$ and the corresponding dyadic cube $c_{\lambda}=\tilde{\lambda}+[0,1]^{d} 2^{-j-1}$. Let $\Lambda$ be the set of $\lambda$ 's such that $j \geq 0$, and $\underline{\Lambda}$ for the whole set $(j \in \mathbb{Z})$.

The correlation (or Gram) matrix of a $\left(\mathcal{S}\left(\mathbb{R}^{d}\right) \rightarrow \mathcal{S}^{\prime}\left(\mathbb{R}^{d}\right)\right)$ continuous operator $A$ is

$$
M_{A}\left(\lambda, \lambda^{\prime}\right)=\left(A\left(\psi_{\lambda}\right) \mid \psi_{\lambda^{\prime}}\right)_{L^{2}}, \quad \lambda, \lambda^{\prime} \in \Lambda
$$




\subsubsection{Wavelet orthonormal basis associated with a pseudo- differential operator.}

Let us define

$$
\Phi_{\lambda}=A_{g}^{-1 / 2}\left(\psi_{\lambda}\right), \quad \lambda \in \Lambda .
$$

We can restate the norm equivalence of Proposition 1.1 as follows: $A_{g}^{-1 / 2}$ is of the form $D \bar{M} D$ with $\bar{M}$ bounded on $l^{2}$ and $D\left(\lambda, \lambda^{\prime}\right)=$ $2^{-j m / 2} \delta_{\lambda, \lambda^{\prime}}$. The important result that we will prove at the beginning of Part 2 is decay of the entries of $\bar{M}$ : we will show that this matrix is "almost diagonal" (in a sense that will be made precise in Definition 2.1 ). This will easily imply that the $\Phi_{\lambda}$ have the following "wavelet-like" decay properties and have an "asymptotic behavior" for large $j$ 's.

Theorem 1.1. Let $m, \gamma>0$, suppose that Hypothesis $\operatorname{HA}(m, \gamma)$ holds and that $A_{g}$ satisfies $\operatorname{HAS}(m, \gamma)$. The $\left\{\Phi_{\lambda}\right\}_{\lambda \in \Lambda}$ defined by (17) form an orthonormal basis of $H_{A_{g}}$ with the following smoothness and localization properties.

$$
\text { If }|s| \leq[m / 2],
$$

$$
\left|\partial^{s} \Phi_{\lambda}(x)\right| \leq \frac{C_{\gamma} 2^{j(d / 2+|s|-m / 2)}}{\left(1+2^{j}|x-\lambda|\right)^{d+\gamma}} .
$$

If $|s|=[m / 2]$,

$$
\left|\partial^{s} \Phi_{\lambda}(x)-\partial^{s} \Phi_{\lambda}(y)\right| \leq \frac{C_{\gamma}|x-y|^{m / 2-|s|} 2^{j d / 2}}{\left(1+2^{j}|x-\lambda|\right)^{d+\gamma}} .
$$

If $[m / 2] \leq|s| \leq[\gamma+m / 2]$,

$$
\left|\partial^{s} \Phi_{\lambda}(x)\right| \leq \frac{C_{\gamma} 2^{j(d / 2+|s|-m / 2)}}{\left(1+2^{j}|x-\lambda|\right)^{d+\gamma+m / 2-|s|}} .
$$

$$
\text { If }|s|=[\gamma+m / 2]
$$

$$
\left|\partial^{s} \Phi_{\lambda}(x)-\partial^{s} \Phi_{\lambda}(y)\right| \leq \frac{C_{\gamma}|x-y|^{\gamma+m / 2-|s|} 2^{j d / 2}}{\left(1+2^{j}|x-\lambda|\right)^{d}} .
$$

The following theorem which describes the asymptotic behaviour of the wavelets when $j \rightarrow+\infty$ shows that in this limit the wavelets are 
the same as wavelets associated with a Selfsimilar Gaussian Process; thus it will allow us to derive the local scaling properties of the process $X$.

Theorem 1.2. The hypotheses are the same as in Theorem 1.1. Let $\left(g_{\lambda}\right)$ be defined by its Fourier transform as follows

$$
\hat{g}_{\lambda}(\xi)=(\sigma(\lambda, \xi))^{-1 / 2} \hat{\psi}_{\lambda}(\xi)
$$

Then for all $\varepsilon$, there exists $J$ such that, for $s \leq m / 2$ and $j \geq J$

$$
\left|\partial^{s} g_{\lambda}(x)-\partial^{s} \Phi_{\lambda}(x)\right| \leq \frac{\varepsilon 2^{(d / 2+|s|-m / 2) j}}{\left(1+2^{j}|x-\lambda|\right)^{d+\gamma}}
$$

1.4.3. REMARKS. Let us now give a few remarks concerning the kind of symbols we consider here and the wavelets we use. First we used nonnegative scales $(j \geq 0)$ for the following reason. If we used all the $\psi_{j, k}^{(l)}$ even for negative and arbitrary large $j$ (and no $\phi_{k}$ ) we would not be able to decompose symbols that depend on $x$ (and then in Part 4, to analyse stochastic processes that have nonstationary increments). In fact when the symbol depends on $x$ and thus presents oscillation at (say) scale $2^{-j_{0}}$, its action on a wavelet indexed by $-j \ll-j_{0}$ does not give a "vaguelette" at scale $2^{-j}$; the function we obtain oscillates too much. Thus the matrix of $A$ in a basis composed of all the $\psi_{j, k}^{(l)}$ (including negative and arbitrary large $j$ ) would not be "almost diagonal".

On the other hand, since we have to use the $\phi(x-k)$ we may not allow the symbol to vanish or to have a pole at 0 ; otherwise it would introduce a singularity at 0 .

\subsection{Regularity of the Elliptic Gaussian Processes.}

In this part $m>d,(l, \alpha) \in \mathbb{N} \times] 0,1]$ is defined by

$$
\frac{m-d}{2}=l+\alpha
$$

Before giving the uniform modulus and the iterated logarithm law of the processes $X_{A}$, let us start with a "global" regularity result which is a straightforward consequence of the wavelet decomposition of $X_{A_{g}}$, 
under hypothesis $\mathrm{HA}(m, \gamma)$ for $A$. Let us recall that a function $f$ belongs to the Besov space $B_{p, q}^{s}$ if

$$
|f|_{s, p, q}:=\|f\|_{L^{p}}+\sum_{|r|=[s]}\left|\partial^{r} f\right|_{\beta, p, q}<\infty
$$

where

$$
\begin{array}{r}
\omega_{p}(g, t)=\sup _{|y| \leq t}\|(g(\cdot+y)-g(\cdot))\|_{L^{p}} \\
\beta=s-[s] \\
|g|_{\beta, p, q}^{q}=\int_{0}^{\infty}\left(\frac{\omega_{p}(g, t)}{t^{\beta}}\right)^{q} \frac{d t}{t}
\end{array}
$$

with the usual modification when $q=\infty$. Let us also recall that Sobolev and Hölder spaces are given by $H^{s}=B_{2,2}^{s}$ and $C^{s}=B_{\infty, \infty}^{s}$.

\subsubsection{Regularity of the process $X_{A_{g}}$.}

Proposition 1.2. If the symbol $\sigma$ satisfies $\operatorname{HA}(m, \gamma)$, then,

i) for each $\Phi \in H_{A_{g}}, \mathcal{A}_{g}\left(X_{A_{g}}, \Phi\right)$ is a well defined random variables of law $\mathcal{N}\left(0,\|\Phi\|_{A_{g}}\right)$;

ii) for each bounded open set $U \subset \mathbb{R}^{d}$,

$$
X_{A_{g}}(x)=\sum_{\lambda \in \Lambda} \Phi_{\lambda}(x) \mathcal{A}_{g}\left(X_{A_{g}}, \Phi_{\lambda}\right)
$$

with uniform convergence of the serie and its derivatives up to order $l$ on $U$.

iii) The above series converges locally in $B_{p, q}^{s}$ when $s<l+\alpha \mathbb{P}$ almost surely.

In dimension $d=1$ and for the fractionnal brownian motion of order $\alpha$, assertion ii) of this proposition is proved in [11]. Note that Besov spaces have also been used by D. Donoho and his collaborators (see [14]) as a particularly convenient setting for wavelet based methods in statistics. 


\subsubsection{Laws of uniform and local moduli of continuity.}

Let us define $d_{i, s}(x, y)\left((x, y) \in \mathbb{R}^{d} \times \mathbb{R}^{d}, i=1,2\right)$ by

$$
\begin{gathered}
d_{1, s}^{2}(x, y)=\mathbb{E}\left[\left(\partial^{s} X_{A}(x)-\partial^{s} X_{A}(y)\right)^{2}\right] \\
(24) \quad d_{2, s}^{2}(x, y)=\mathbb{E}\left[\left(\partial^{s} X_{A}(x)-2 \partial^{s} X_{A}\left(\frac{x+y}{2}\right)+\partial^{s} X_{A}(y)\right)^{2}\right]
\end{gathered}
$$

Recall that $m-d=2(l+\alpha), l \in \mathbb{N}, 0<\alpha \leq 1$. For any multi-index $s$ of lenght $|s|=l$ we define,

1) when $\alpha=1$,

$$
c_{2, s}(y)=\limsup _{x \rightarrow y} \frac{d_{2, s}(x, y)}{|x-y|}
$$

and

$$
c_{1, s}(y)=\limsup _{x \rightarrow y} \frac{d_{1, s}(x, y)}{|x-y| \sqrt{\log \left(|x-y|^{-1}\right)}} .
$$

2) When $\alpha<1$,

$$
c_{1, s}(y)=\limsup _{x \rightarrow y} \frac{d_{1, s}(x, y)}{|x-y|^{\alpha}}
$$

Lemma 1.2. Under the hypothesis $\mathrm{HA}(m, \gamma)$ and if $|s|=l$, the functions $c_{1, s}, c_{2, s}$ belong to $C^{\left(\varepsilon-\varepsilon^{\prime}\right) / 2}\left(\mathbb{R}^{d}\right)$.

Let us now set, when $D$ is a bounded open subset of $\mathbb{R}^{d}$,

$$
c_{i, s, D}=\sup _{y \in D} c_{i, s}(y) .
$$

We can express the main result of this paragraph, where we use the notation, for $r$ small enough

$$
L(r)=\log \left(\frac{1}{r}\right), \quad L_{k}(r)=\log \circ \cdots \circ \log \left(\frac{1}{r}\right), \quad k \text { times }
$$


Theorem 1.3. Under the hypothesis $\operatorname{HA}(m, \gamma)$, if $m>d, s \in \mathbb{N}^{d}$, $|s|=l$,

i) law of the uniform modulus:

- when $\alpha<1$,

$$
\limsup _{x, y \in D,|x-y| \rightarrow 0} \frac{\left|\partial^{s} X_{A}(x)-\partial^{s} X_{A}(y)\right|}{|x-y|^{\alpha} \sqrt{L\left(|x-y|^{-1}\right)}}=\sqrt{2 d} c_{1, s, D},
$$

$\mathbb{P}$ almost everywhere,

- when $\alpha=1$,

$$
\begin{aligned}
& \limsup _{x, y \in D,|x-y| \rightarrow 0} \frac{\left|\partial^{s} X_{A}(x)-2 \partial^{s} X_{A}\left(\frac{x+y}{2}\right)+\partial^{s} X_{A}(y)\right|}{|x-y| \sqrt{L\left(|x-y|^{-1}\right)}} \\
& =\sqrt{2 d} c_{2, s, D},
\end{aligned}
$$

$\mathbb{P}$ almost everywhere,

- and

$$
\limsup _{x, y \in D,|x-y| \rightarrow 0} \frac{\left|\partial^{s} X_{A}(x)-\partial^{s} X_{A}(y)\right|}{|x-y| L\left(|x-y|^{-1}\right)}=\sqrt{2 d} c_{1, s, D}
$$

$\mathbb{P}$ almost everywhere.

ii) Law of the iterated logarithm:

- when $\alpha<1$, for all $y \in \mathbb{R}^{d}$,

$$
\limsup _{x \rightarrow y} \frac{\left|\partial^{s} X_{A}(x)-\partial^{s} X_{A}(y)\right|}{|x-y|^{\alpha} \sqrt{L_{2}\left(|x-y|^{-1}\right)}}=\sqrt{2} c_{1, s}(y)
$$

$\mathbb{P}$ almost everywhere,

- when $\alpha=1$, for all $y \in \mathbb{R}^{d}$,

(32) $\limsup _{x \rightarrow y} \frac{\left|\partial^{s} X_{A}(x)-\partial^{s} X_{A}(y)\right|}{|x-y| \sqrt{L\left(|x-y|^{-1}\right)} \sqrt{L_{3}\left(|x-y|^{-1}\right)}}=\sqrt{2} c_{1, s}(y)$,

$\mathbb{P}$ almost everywhere. 
Note that the law of the iterated logarithm may be used to identify the "principal part" of the symbol, when it exists. If we assume that

$$
\sigma(x, \xi)=a_{x}\left(\frac{\xi}{|\xi|}\right)|\xi|^{m}+o\left(|\xi|^{m}\right)
$$

we will obtain later, see (60), in the case $\alpha<1$, the very explicit formula

$$
c_{1, s}^{2}(y)=\sup _{|v|=1} \int_{\mathbb{R}^{d}} 4 \frac{\sin ^{2}(v \xi / 2)}{|\xi|^{d+2 \alpha} a_{y}(\xi /|\xi|)} d \xi,
$$

(31) shows the precise relationship between the "principal part" of the symbol $\sigma$ and the exact local modulus of continuity of the process $X$.

Theorem 1.3 is proved in Section 4. The main idea is first to get the results for the modified process $X_{A_{g}}$, using its wavelet decomposition (see Theorem 1.1) and then to transfer the regularity properties of $X_{A_{g}}$ to $X_{A}$, as a consequence of Proposition 1.1.

1.5.3. REMARKs. When comparing (26) with (27), or (28) with (30), or (31) with (32) it appears that the case $\alpha=1$ is critical. In fact this goes back to formula (18) which for $\alpha=1,|s|=l$ gives

$$
\partial^{s} \Phi_{\lambda}(x)-\partial^{s} \Phi_{\lambda}(y) \simeq C_{\gamma}|x-y|, \quad|x-y| \rightarrow 0,
$$

with $C_{\gamma}$ independent of the scale $j$ of $\lambda$. Assuming $\Phi_{\lambda}$ is supported by the dyadic cube $c_{\lambda}$, with center $\lambda$ and sidelenght $2^{-j}$, we would have when $l=0, \alpha=1,|y-x| \leq 2^{-n}$

$$
\frac{\left|\partial^{s} X(x)-\partial^{s} X(y)\right|}{|x-y|} \simeq C \sum_{\lambda \in \Lambda \cap E, j_{\lambda} \leq n} \mathbf{1}_{\left\{c_{\lambda}(x)\right\}} \xi_{\lambda}
$$

(30) and (35) will be obtained by studying large deviations for sums of normal random variable indexed by a tree in Section 3 .

On the other hand, formula (18) implies that in the case $\alpha<1$ the uniform and local moduli can be studied with sums restricted to the scales $j$ near $\log _{2}(|x-y|)$, so that the proofs of (28) and (31) are close to the proofs of [3]. 


\subsubsection{Comparison with known results.}

When the symbol $\sigma$ is a function of $\xi$ only, and $m>d$ the process $X_{A}$ has stationary increments. Let us then define $\rho(h)$ near the origin by

$$
\rho(|x-y|)=d_{s}(x, y) .
$$

Kono in [26] assumes that $\rho^{2}$ is concave and increasing near 0. Formula (27) shows that, even in the stationary case, none of these two conditions needs to be satisfied. In dimension 1, Marcus [29, Theorem 3.8] obtains the modulus of continuity under wider asumptions than Kono, which however do not include the critical case $\alpha=1$ that we consider.

The results of Lemma 1.2 imply the hypotheses of Theorem 2.10 in [15] which asserts the existence of a bounded random variable $K(\omega)$ such that if $\alpha<1$

$$
\left|\partial^{s} X_{A}(x)-\partial^{s} X_{A}(y)\right| \leq K(\omega)|x-y|^{\alpha} \log \left(\frac{1}{|x-y|}\right)^{1 / 2}
$$

$\mathbb{P}$ almost surely, and when $\alpha=1$

$$
\left|\partial^{s} X_{A}(x)-\partial^{s} X_{A}(y)\right| \leq K(\omega)|x-y| \log \left(\frac{1}{|x-y|}\right),
$$

$\mathbb{P}$ almost surely, which is clearly less accurate than Theorem 1.3 in the elliptic context. If $A=\prod_{i=1}^{d}\left(-\Delta+c_{i}^{2}\right)$, the results of Proposition 1.1 are proved in [8]. If $A$ is differential with $C^{\infty}$ coefficients, it is proved in [1] that

$$
\mathbb{P}\left(X_{A} \in H_{\mathrm{loc}}^{l-d-\varepsilon}\right)=1, \quad \text { for all } \varepsilon>0 .
$$

\subsection{Local scaling properties of Elliptic Gaussian Processes.}

In this paragraph, we suppose that the symbol $\sigma$ satisfies $H A$ $(m, \gamma)$. We show that the process $X_{A}$ satisfies some local scaling property when its symbol $\sigma$ admits a "principal part" (which is positively homogeneous).

We will distinguish the two cases $\alpha<1$ and $\alpha=1$ ( $l$ and $\alpha$ are defined by (22)). 
Definition 1.2. Suppose that $0<\alpha<1$. The E.G.P. $X_{A}$ is Asymptotically Self Similar (L.A.S.S.) of order $(\alpha, l)$ if

$$
\lim _{\rho \rightarrow 0^{+}} \operatorname{Law}\left\{\frac{(-\Delta)^{l / 2} X_{A}(x+\rho u)-(-\Delta)^{l / 2} X_{A}(x)}{\rho^{\alpha}}, u \in \mathbb{R}^{d}\right\},
$$

exists for every $x$ and is not trivial. $X_{A}$ is Weakly Asymptotically Self Similar (W.L.A.S.S.) of order $(\alpha, l)$ if for every $x$, we can find a sequence $\left(\rho_{n}\right) \longrightarrow 0^{+}$such that

$$
\frac{(-\Delta)^{l / 2} X_{A}\left(x+\rho_{n} u\right)-(-\Delta)^{l / 2} X_{A}(x)}{\rho_{n}^{\alpha}}, \quad u \in \mathbb{R}^{d},
$$

converges in law to a non trivial limit.

When $m=d+2 \alpha, 0<\alpha<1(l=0)$ the following theorem characterizes the L.A.S.S. property. The general case is similar, after $l$ differentiations.

Theorem 1.4. If the symbol $\sigma$ satisfies $\mathrm{HA}(d+2 \alpha, \gamma)$ for $0<\alpha<1$, the following assertions are equivalent.

i) $X_{A}$ is a L.A.S.S. of order $(\alpha, 0)$.

ii) For all $x_{0} \in \mathbb{R}^{d}$,

$$
\lim _{\rho \rightarrow \infty} \frac{\sigma\left(x_{0}, \rho \xi\right)}{\rho^{d+2 \alpha}}=\theta_{x_{0}}(\xi),
$$

exists, $\theta$ is an $(d+2 \alpha)$-homogeneous non trivial symbol and $\theta \in S_{\infty, 0}^{d+2 \alpha}$.

iii) For all $x_{0} \in \mathbb{R}^{d}$,

$$
\lim _{h \rightarrow 0^{+}} \mathbb{E}\left[\frac{\left(X_{A}\left(x_{0}+h u\right)-X_{A}\left(x_{0}\right)\right)^{2}}{h^{2 \alpha}}\right]=c_{x_{0}}^{2}(u),
$$

exists and the function $c_{x_{0}}$ is an $\alpha$-homogeneous non trivial function.

We now consider the critical case $\alpha=1$.

Definition 1.3. Suppose that $\alpha=1$. The E.G.P. $X_{A}$ belongs to the weakly Locally Critical (W.L.C.) class of order $l$ if for every $x$, we can find a sequence $\left(\rho_{n}\right)$ which goes to $0^{+}$such that

$$
\frac{(-\Delta)^{l / 2} X_{A}\left(x+\rho_{n} u\right)-(-\Delta)^{l / 2} X_{A}(x)}{\rho_{n} \sqrt{\log \left(1 / \rho_{n}\right)}}, \quad u \in \mathbb{R}^{d},
$$


converges in law to the process $\left\{(G, u), u \in \mathbb{R}^{d}\right\}$ with $G$ a gaussian random variable on $\mathbb{R}^{d}$. It belongs to the Locally Critical (L.C.) class of order $(\alpha, l)$ if for every $x$ there exists $G$ a gaussian random variable on $\mathbb{R}^{d}$ such that

$$
\begin{array}{r}
\lim _{\rho \rightarrow 0^{+}} \operatorname{Law}\left\{\frac{(-\Delta)^{l / 2} X_{A}(x+\rho u)-(-\Delta)^{l / 2} X_{A}(x)}{\rho \sqrt{\log (1 / \rho)}}, u \in \mathbb{R}^{d}\right\} \\
=\operatorname{Law}\left\{(G, u), u \in \mathbb{R}^{d}\right\} .
\end{array}
$$

for every $x$.

The following theorem gives a characterization of the Locally Critical class.

Theorem 1.5. If the symbol $\sigma$ satisfies $\mathrm{HA}(d+2, \gamma)$, the following assertions are equivalent.

i) $X_{A}$ is Locally Critical of order 0 .

ii) For all $x_{0} \in \mathbb{R}^{d}$,

$$
\lim _{\rho \rightarrow \infty} \frac{\sigma\left(x_{0}, \rho \xi\right)}{\rho^{d+2}}=\theta_{x_{0}}(\xi)
$$

exists and $\theta_{x_{0}}$ is an $(d+2)$-homogeneous and non trivial symbol which belongs to $S_{\infty, 0}^{d+2}$.

iii) For all $x_{0} \in \mathbb{R}^{d}$

$$
\lim _{h \rightarrow 0^{+}} \mathbb{E}\left[\frac{\left(X_{A}(x+h u)-X_{A}(x)\right)^{2}}{h^{2} \log (1 / h)}\right]=c_{x_{0}}^{2}(u),
$$

exists and the function $c_{x_{0}}$ is an 1-homogeneous non trivial function.

Condition (38) or (40) means that the symbol has the following asymptotic behavior

$$
\sigma(x, \xi)=h(x) F\left(\frac{\xi}{|\xi|}\right)|\xi|^{m}+o\left(|\xi|^{m}\right)
$$

it excludes symbols which have some slow oscillations at high frequencies like

$$
\sigma(x, \xi)=|\xi|^{m}\left(1+\sin ^{2}\left(\log \left(1+|\xi|^{2}\right)\right)\right)
$$


such symbols give rise to processes which belongs only to the above weak classes. More precisely, let us consider a symbol $\sigma$ such that

$$
\sigma(\xi)=\sigma_{0}(\xi) f(\xi)
$$

where the symbol $\sigma_{0}$ is supposed to satisfy one of the equivalent conditions of Theorem 1.4. Let $X_{0}$ be the gaussian process associated to $\sigma_{0}$, wich belongs to the L.A.S.S. class of order $(\alpha, 0)$.

Proposition 1.3. Let $f$ be an even and $C^{\infty}$ function on $\mathbb{R}^{d}$ such that the operator of symbol $1 / f$ is positive definite, $1 / f$ belongs to $L_{\mathrm{loc}}^{1}$ and, for all $s \in \mathbb{N}^{d}$ there exists $C_{s}>0$ such that

$$
\left|\partial^{s} f(\xi)\right| \leq C_{s}(1+|\xi|)^{-|s|}
$$

The gaussian process associated with $\sigma$ in (41) belongs to the W.L.A. S.S. class of order $(\alpha, 0)$. Moreover $X$ belongs to the L.A.S.S. class of order $(\alpha, 0)$ if and only if $\lim _{|\xi| \rightarrow \infty} f(\xi)$ exists and does not vanish.

The proofs of the local scaling properties are given in Part 5.

\subsection{Complements.}

We now consider two interesting cases that do not fit strictly speaking in the framework of Elliptic Gaussian Random Fields (E.G.R.F.), but can nonetheless be studied by the methods introduced in this paper. First we will consider the Generalized Gaussian Processes where the order of $A$ is less than $d / 2$; in that case the corresponding process is no more a function but a distribution. The second one is a Fractional Brownian motion of nonconstant order that we will define in (42). It will not be an E.G.R.F. but we will see that there is also a wavelet basis "adapted" to this process so that the technique we developed will immediately yield its regularity and scaling properties.

\subsubsection{Extension to Generalized Gaussian Processes.}

For the sake of simplicity we will consider only two cases which are important in applications: the " $1 / f$ noise" which is used in signal 
analysis (see [28]) and the "free field" which is used in quantum field theory (see [6] and [7]).

We consider in dimension 1 the operator $A=(-\Delta)^{1 / 2}$; the process $X_{A}$ is no longer a random function, but a random distribution, i.e. a Generalized Gaussian Process (G.G.P.) which is called the $1 / f$ noise (see [33]), because of its spectral function.

Let now $d \geq 2, q \in \mathbb{R}^{+}$and $A=-\Delta+q^{2}$. The process associated with $A$ is by definition the free field of mass $q$.

In both situations, let us define a "truncated process" as follows

$$
X_{n}(x)=\sum_{\lambda \subset E, j_{\lambda} \leq n} \Phi_{\lambda}(x) \xi_{\lambda}
$$

with $E=(0,1)^{d}$.

Theorem 1.6. For every $d \in \mathbb{N}^{*}$ there exists $C_{d}>0$ such that,

1) if $d=1$ or 2 ,

$$
\limsup _{n \rightarrow \infty} \frac{1}{n} \sup _{x \in E}\left|X_{n}(x)\right|=C_{d}, \quad \mathbb{P} \text { almost everywhere, }
$$

2) if $d>2$,

$$
\limsup _{n \rightarrow \infty} \sqrt{\frac{2^{-n(d-1)}}{n}} \sup _{x \in E}\left|X_{n}(x)\right|=C_{d}, \quad \mathbb{P} \text { almost everywhere. }
$$

This result, which will be proved in Section 5, shows the rate of divergence in the space of bounded functions of the processes $X_{n}$ which are approximations of $X$ in the distribution sense. If $d=1$, we see that $X_{n}$ diverges very slowly. This shows why the fact that $X$ is not a function but a distribution is hard to detect on numerical simulations, see [33]. In field theory [8] the difficulties of the renormalization increase with $d$ (If $d=4,\left|X_{n}\right|_{\infty}$ diverges like $\sqrt{n} 2^{3 n / 2}$ which shows one of the reasons of the difficulty of $P \Phi_{4}$ theory). We must also mention the connected work [6] where the renormalisation of sums like

$$
X_{n}(x)=\sum_{j_{\lambda} \leq n} \phi_{\lambda}(x) \xi_{\lambda} 2^{|\lambda| d / 2}
$$

is studied when the $\xi_{\lambda}$ are Rademacher or Gaussian random variables $\xi_{\lambda}$, and $\phi_{\lambda}(x)$ is the indicatrix function of a dyadic cell $\lambda$. 


\subsubsection{Multifractional Brownian Motion.}

Let us state the definition we adopt for the Multifractional Brownian Motion which extends (8).

Definition 1.4. Let $a \in C^{r}\left(\mathbb{R}^{d},(0,1)\right)$ for some $r>\sup a(x)$ and $W$ a white noise. The Multifractional Brownian motion of order $a(x)$ is defined by

$$
B_{a}(x)=\int \frac{e^{i x \xi}-1}{|\xi|^{a(x)+d / 2}} d \overline{\hat{W}}(\xi)
$$

The function $C: \mathbb{R}^{d} \rightarrow \mathbb{R}$ defined by

$$
C^{2}(x)=\int \frac{1-\cos ^{2}(x \nu)}{|\nu|^{d+2 a(x)}} d \nu
$$

belongs to $C^{r}\left(\mathbb{R}^{d}\right)$ and

$$
\mathbb{E}\left(\left|B_{a}(x+h)-B_{a}(x)\right|^{2}\right)=C^{2}(x)|h|^{2 a(x)}+\mathrm{o}(h) .
$$

In order to obtain a wavelet decomposition of $B_{a}$, one uses the following decomposition of the white noise on the Fourier transforms of the Littlewood-Paley wavelet basis

$$
d W(\xi)=\sum \eta_{\lambda} \hat{\psi}_{\lambda}(\xi) d \xi
$$

where the $\eta_{\lambda}$ are i.i.d. standard gaussian; if

$$
\omega_{\lambda}(x)=\int \frac{e^{i x \xi}-1}{|\xi|^{a(x)+d / 2}} \hat{\bar{\psi}}_{\lambda}(\xi) d \xi
$$

then

$$
B_{a}(x)=\sum \eta_{\lambda} \omega_{\lambda}(x)
$$

and the following result will be a consequence of "vaguelettes" decay estimates for the $\omega_{\lambda}$. In this part we define $a_{E}=\inf _{x \in E} a(x), C_{E}=$ $\sup _{x \in a^{-1}\left(a_{E}\right) \cap \bar{E}} C(x)$, when $E$ is a bounded open set. 
Theorem 1.7. Let $E$ be an open bounded set. The Multifractional Brownian motion $B_{a}$ satisfies the following law of the uniform modulus of continuity

$$
\limsup _{x, y \in E,|x-y| \rightarrow 0} \frac{\left|B_{a}(x)-B_{a}(y)\right|}{|x-y|^{a_{E}} \sqrt{\log (1 /|x-y|)}}=C_{E} \sqrt{2 d},
$$

$\mathbb{P}$ almost everywhere, and the law of the iterated logarithm, for all $y \in$ $\mathbb{R}^{d}$

$$
\limsup _{x \rightarrow y} \frac{\left|B_{a}(x)-B_{a}(y)\right|}{|x-y|^{a(y)} \sqrt{\log \log (1 /|x-y|)}}=\sqrt{2} C(x),
$$

$\mathbb{P}$ almost everywhere. Furthermore, $B_{a}$ is Asymptotically Self Similar of order $a\left(x_{0}\right)$ at $x_{0}$; i.e.

$$
\lim _{\rho \rightarrow 0^{+}} \operatorname{Law}\left\{\frac{B\left(x_{0}+\rho u\right)-B\left(x_{0}\right)}{\rho^{a\left(x_{0}\right)}}, u \in \mathbb{R}^{d}\right\}=\operatorname{Law}\left\{B_{a\left(x_{0}\right)}\right\} .
$$

The reader can check that the same analysis would work after introducing in (42) a directional dependancy $S(\xi)$.

\section{Wavelets and Elliptic Operators.}

In this part we will construct the wavelet basis of $H_{A}$ and prove Theorems 1.1 and 1.2 under regularity hypotheses on the symbol $\sigma$. We will also prove the equivalence in law stated in Proposition 1.1. This opens the way to Theorem 1.3 (proved in Section 4) which gives the uniform and local moduli of continuity of the process.

\subsection{Wavelet matrices of pseudodifferential elliptic operators.}

The basic idea here is not to work directly on the operator itself but rather on the infinite matrix of its coefficients on a wavelet basis. We will show that the matrices of the pseudodifferential operators we consider and of their inverses are of the form $D M D$ where $D$ is a diagonal matrix in a wavelet basis whereas $M$ and $M^{-1}$ are "almost diagonal" in the following sense (see [30]). 
Definition 2.1. 1) A matrix $M\left(\lambda, \lambda^{\prime}\right)$ belongs to the algebra $\mathcal{M}^{\gamma}$ if

$$
\left|M\left(\lambda, \lambda^{\prime}\right)\right| \leq C \omega_{\gamma}\left(\lambda, \lambda^{\prime}\right)
$$

where

$$
\omega_{\gamma}\left(\lambda, \lambda^{\prime}\right)=\frac{2^{-(d / 2+\gamma)\left|j-j^{\prime}\right|}}{\left(1+\left|j-j^{\prime}\right|^{2}\right)\left(1+2^{\inf \left(j, j^{\prime}\right)}\left|\lambda-\lambda^{\prime}\right|\right)^{d+\gamma}} .
$$

2) A matrix $M\left(\lambda, \lambda^{\prime}\right)$ belongs to $\mathcal{M}^{\gamma, m}(m \in \mathbb{R})$ if $M=D \bar{M} D$ with $\bar{M} \in \mathcal{M}^{\gamma}$ and $D\left(\lambda, \lambda^{\prime}\right)=2^{j m / 2} \delta_{\lambda, \lambda^{\prime}}$.

For operators, we have the corresponding classes.

Definition 2.2. An operator $A$ belongs to $\mathrm{OP}\left(\mathcal{M}^{\gamma, m}\right)$ if its matrix $M_{\lambda, \lambda^{\prime}}=\left\langle A\left(\psi_{\lambda}\right) \mid \psi_{\lambda^{\prime}}\right\rangle$ in the "Littlewood-Paley" wavelet basis belongs to $\mathcal{M}^{\gamma, m}$.

The following Proposition shows that the class of symbols considered here is related to the class of matrices just defined. Therefore let

$$
\delta(m, \gamma):=\min \left\{[\gamma]+1, \frac{m}{2}\right\}
$$

Proposition 2.1. If the symbol $\sigma$ satisfies $\mathrm{HA}(m, \gamma)$, then $A \in$ $\mathrm{OP}\left(\mathcal{M}^{\delta, m}\right)$, for all $\delta<\delta(m, \gamma)$.

The following theorem asserts a kind of symbolic calculus for the operators we consider.

Theorem 2.1. If $A$ satisfies $\operatorname{HAS}(m, \gamma), A^{-1}$ belongs to OP $\left(\mathcal{M}^{\delta,-m}\right)$, for all $\delta<\delta(m, \gamma)$.

We now prove Proposition 2.1. Let $r=[\gamma]+d+1, \delta=\delta(m, \gamma)$. We know that $\sigma$ belongs to $S_{r}^{m}$. Denote by $M_{\lambda, \lambda^{\prime}}$ the entries of $M_{A}$, the matrix of $A$ in $\left(\psi_{\lambda}\right)_{j_{\lambda} \geq 0}$. Since $A$ is self-adjoint, we consider only the case $j^{\prime} \geq j$;

$$
\begin{aligned}
M_{\lambda, \lambda^{\prime}} & =\iint \sigma(x, \xi) e^{i x \xi} \hat{\psi}_{\lambda}(\xi) \psi_{\lambda^{\prime}}(x) d x d \xi \\
& =2^{d\left(j^{\prime}-j\right) / 2} \iint \sigma(x, \xi) e^{i(x-\lambda) \xi} \hat{\psi}^{(l)}\left(\frac{\xi}{2^{j}}\right) \psi^{\left(l^{\prime}\right)}\left(2^{j^{\prime}} x-k^{\prime}\right) d x d \xi
\end{aligned}
$$


and thus

$$
\begin{aligned}
M_{\lambda, \lambda^{\prime}}=2^{-d\left(j^{\prime}-j\right) / 2} \iint \sigma & \left(\frac{x}{2^{j^{\prime}}}+\lambda^{\prime}, 2^{j} \xi\right) \\
& \cdot e^{i\left(x / 2^{j^{\prime}}+\lambda^{\prime}-\lambda\right) 2^{j} \xi} \hat{\psi}^{(l)}(\xi) \psi^{\left(l^{\prime}\right)}(x) d x d \xi .
\end{aligned}
$$

Since the functions $\psi^{(l)}$ have fast decay, there exists a $K>0$ such that, for all $l \in\{0,1\}^{d}$ and $\rho \geq 1 / 2$,

$$
\int_{|x|>\rho / 2 d}\left|\psi^{(l)}(x)\right| d x \leq K \rho^{-r} .
$$

We distinguish two more cases.

Case 1. $l^{\prime} \neq 0$ and $2^{j}\left|\lambda-\lambda^{\prime}\right|<1 / 2$.

The function $F\left(\lambda, \lambda^{\prime}, x, \xi\right)=\sigma\left(x+\lambda^{\prime}, \xi\right) e^{i\left(x+\lambda^{\prime}-\lambda\right) \xi}$ satisfies estimates (6) and (7). Thus, since $\psi^{\left(l^{\prime}\right)}$ has a vanishing integral

$$
\begin{gathered}
M_{\lambda, \lambda^{\prime}}=2^{-d\left(j^{\prime}-j\right) / 2} \iint F\left(\lambda, \lambda^{\prime}, 2^{-j^{\prime}} x, 2^{j} \xi\right) \hat{\psi}^{(l)}(\xi) \psi^{\left(l^{\prime}\right)}(x) d x d \xi \\
=2^{-d\left(j^{\prime}-j\right) / 2} \iint\left(F\left(\lambda, \lambda^{\prime}, 2^{-j^{\prime}} x, 2^{j} \xi\right)-F\left(\lambda, \lambda^{\prime}, 0,2^{j} \xi\right)\right) \\
\cdot \hat{\psi}^{(l)}(\xi) \psi^{\left(l^{\prime}\right)}(x) d x d \xi
\end{gathered}
$$

so that

$$
\begin{aligned}
\left|M_{\lambda, \lambda^{\prime}}\right| \leq & C_{0} 2^{-d\left(j^{\prime}-j\right) / 2} \\
& \cdot \iint\left|2^{-j^{\prime}} x\right|^{\varepsilon}\left(1+2^{j}|\xi|\right)^{m+\varepsilon}\left|\hat{\psi}^{(l)}(\xi)\right|\left|\psi^{\left(l^{\prime}\right)}(x)\right| d x d \xi \\
\leq & C_{1} 2^{-(d / 2+\varepsilon+m / 2)\left(j^{\prime}-j\right)} 2^{m j / 2} 2^{m j^{\prime} / 2} \\
\leq & C_{2} \omega_{\delta(m, \gamma)}\left(\lambda, \lambda^{\prime}\right) .
\end{aligned}
$$

Case 2. $2^{j}\left|\lambda-\lambda^{\prime}\right| \geq 1 / 2$.

Let

$$
\begin{gathered}
J:=2^{-d\left(j^{\prime}-j\right) / 2} \iint_{|x|>\rho / 2 d} \sigma\left(\frac{x}{2^{j^{\prime}}}+\lambda^{\prime}, 2^{j} \xi\right) e^{i\left(x / 2^{j^{\prime}}+\lambda^{\prime}-\lambda\right) 2^{j} \xi} \\
\cdot \hat{\psi}^{(l)}(\xi) \psi^{\left(l^{\prime}\right)}(x) d x d \xi .
\end{gathered}
$$


Using (50) and hypothesis HA $(m, \gamma)$, we have

$$
\begin{aligned}
|J| & \leq C_{3} 2^{-d\left(j^{\prime}-j\right)} \iint_{|x|>\rho / 2 d}\left(1+2^{j}|\xi|\right)^{m}\left|\hat{\psi}^{(l)}(\xi)\right|\left|\psi^{\left(l^{\prime}\right)}(x)\right| d x d \xi \\
& \leq C_{4} 2^{-d\left(j^{\prime}-j\right) / 2+m j} \rho^{-r}
\end{aligned}
$$

if $\rho \geq 1 / 2$. With $\rho=2^{j^{\prime}}\left|\lambda^{\prime}-\lambda\right|$ we obtain

$$
|J| \leq C_{4} \frac{2^{-d\left(j^{\prime}-j\right) / 2+m j}}{2^{r j^{\prime}}\left|\lambda^{\prime}-\lambda\right|^{r}}=C_{4} 2^{m\left(j+j^{\prime}\right) / 2} \frac{2^{-(d / 2+m / 2+r)\left(j^{\prime}-j\right)}}{\left(2^{j}\left|\lambda^{\prime}-\lambda\right|\right)^{r}} .
$$

The result will be achieved if we get a similar bound for

$$
\begin{gathered}
\tilde{M}_{\lambda, \lambda^{\prime}}=2^{-d\left(j^{\prime}-j\right) / 2} \iint_{|x| \leq 2^{j^{\prime}}\left|\lambda^{\prime}-\lambda\right| / 2 d} \sigma\left(\frac{x}{2^{j^{\prime}}}+\lambda^{\prime}, 2^{j} \xi\right) e^{i\left(x / 2^{j^{\prime}}+\lambda^{\prime}-\lambda\right) 2^{j} \xi} \\
\cdot \hat{\psi}^{(l)}(\xi) \psi^{\left(l^{\prime}\right)}(x) d x d \xi .
\end{gathered}
$$

In fact there exists a coordinate direction, say the $k^{t h}$ one, such that

$$
\left|\lambda_{k}^{\prime}-\lambda_{k}\right| \geq \frac{1}{d}\left|\lambda^{\prime}-\lambda\right|
$$

Integrating by parts $r$ times in the direction $k$, we get

$$
\begin{aligned}
& \tilde{M}_{\lambda, \lambda^{\prime}}= 2^{-d\left(j^{\prime}-j\right) / 2} \\
& \cdot \iint_{|x| \leq 2^{j^{\prime}}\left|\lambda^{\prime}-\lambda\right| / 2 d} \\
& \frac{e^{i\left(x / 2^{j^{\prime}}+\lambda^{\prime}-\lambda\right) 2^{j} \xi}}{\left(i 2^{j}\left(\frac{x_{k}}{2^{j^{\prime}}}+\lambda_{k}^{\prime}-\lambda_{k}\right)\right)^{r}} \\
& \cdot \partial_{\xi_{k}}^{r}\left(\sigma\left(\frac{x}{2^{j^{\prime}}}+\lambda^{\prime}, 2^{j} \xi\right) \hat{\psi}^{(l)}(\xi)\right) \psi^{\left(l^{\prime}\right)}(x) d x d \xi .
\end{aligned}
$$

In the domain of integration, $\left|x_{k} / 2^{j^{\prime}}+\lambda_{k}^{\prime}-\lambda_{k}\right| \geq\left|\left(\lambda-\lambda^{\prime}\right) /(2 d)\right|$ so that

$$
\begin{aligned}
\left|\tilde{M}_{\lambda, \lambda^{\prime}}\right| \leq & C_{5} \frac{2^{-d\left(j^{\prime}-j\right) / 2}}{\left(2^{j}\left|\lambda-\lambda^{\prime}\right|\right)^{r}} \\
& \cdot \iint\left|\partial_{\xi_{k}}^{r}\left(\sigma\left(\frac{x}{2^{j^{\prime}}}+\lambda^{\prime}, 2^{j} \xi\right) \hat{\psi}^{(l)}(\xi)\right) \psi^{\left(l^{\prime}\right)}(x)\right| d x d \xi \\
\leq & C_{6} \frac{2^{-d\left(j^{\prime}-j\right) / 2}}{\left(1+2^{j}\left|\lambda-\lambda^{\prime}\right|\right)^{r}} 2^{m j} \int\left|\psi_{l^{\prime}}(x)\right| d x \\
\leq & C_{7} 2^{m j / 2} 2^{m j^{\prime} / 2} \frac{2^{-(d / 2+m / 2)\left(j^{\prime}-j\right)}}{\left(2^{j}\left|\lambda-\lambda^{\prime}\right|\right)^{r}} .
\end{aligned}
$$


Observe that if $2^{j}\left|\lambda-\lambda^{\prime}\right|<1 / 2$ and $l^{\prime}=0$ we have necessarily $j^{\prime}=j=0$ and thus $\left|k+l / 2-k^{\prime}\right|<1 / 2$, which gives $\lambda=\lambda^{\prime}$; hence Proposition 2.1 .

\subsection{Construction of the wavelets.}

In all this subsection we suppose that $\operatorname{HAS}(m, \gamma)$ holds and we will construct the basis $\left(\Phi_{\lambda}\right)$ under this hypothesis. We define $\Phi_{\lambda}=$ $A^{-1 / 2}\left(\psi_{\lambda}\right)$, where we can use for instance Kato's formula to define $A^{-1 / 2}$

$$
A^{-1 / 2}=\frac{2}{\pi} \int_{0}^{\infty} \frac{d t}{t^{2} \mathrm{Id}+A} .
$$

The fact that the $\left(\Phi_{\lambda}\right)$ form an orthonormal basis of $H_{A}$ is just an algebraic computation since the definition of $A^{-1 / 2}$ is such that $A^{-1 / 2}$ is a positive selfadjoint operator satisfying $A^{-1 / 2} \circ A^{-1 / 2}=A^{-1}$.

Let us recall that a family of functions $\left(f_{\lambda}\right)_{\Lambda}$ is a system of $\delta$ vaguelettes if and only if the matrix of the family in any wavelet basis (with regularity strictly larger than $\delta$ ) belongs to $\mathcal{M}^{\delta}$, see [30].

Proposition 2.2. We have $\Phi_{\lambda}=2^{-m j / 2} \zeta_{\lambda}$ where $\zeta_{\lambda}$ are $\delta$-vaguelettes for every $\delta<\delta(m, \gamma)$.

Let us sketch the proof of Proposition 2.2. Let $t \geq 0$. We define $H(t)$ as the completion of $\mathcal{D}\left(\mathbb{R}^{d}\right)$ for the norm

$$
\|u\|_{H(t)}^{2}=\left\langle\left(t^{2} \mathrm{Id}+A\right) u \mid u\right\rangle .
$$

Lemma 2.1. Wavelets are an unconditional basis of $H(t)$ and the following norm equivalences (uniform in $t$ ) hold

$$
\|u\|_{H(t)}^{2} \sim\|u\|_{H^{m}}^{2}+t^{2}\|u\|_{L^{2}}^{2} \sim \sum_{\lambda}\left|U_{\lambda}\right|^{2} \theta_{\lambda}^{2}
$$

where $U_{\lambda}$ are the wavelet coefficients of $u$ and $\theta_{\lambda}=\sqrt{t^{2}+2^{m j}}$.

The first equivalence is nothing but the asumption $H 1$ on $A$, and the second comes from the wavelet characterization of $H^{s}$ (see [30]). 
Proposition 2.3. The following decomposition holds

$$
t^{2} \mathrm{Id}+M=D^{\prime} \bar{N} D^{\prime},
$$

where $D^{\prime}$ is diagonal on the $L^{2}$-orthonormal (Littlewood-Paley) wavelet basis, $D^{\prime}=\operatorname{Diag}\left(\theta_{\lambda}\right)$ and for all $\delta<\delta(m, \gamma), \bar{N}$ and $\bar{N}^{-1}$ belongs to $\mathcal{M}^{\delta}$ (uniformly in $\left.t\right)$.

Let us admit this proposition for now and see why Proposition 2.2 is a consequence of Proposition 2.3. Using the definition of $A^{-1 / 2}$, we have the following estimate for the matrix coefficients of $A^{-1 / 2}$

$$
\left|M_{\lambda, \lambda^{\prime}}^{-1 / 2}\right| \leq \frac{2}{\pi} \int_{0}^{\infty} \frac{1}{\sqrt{t^{2}+2^{m j}}}\left|\omega\left(\lambda, \lambda^{\prime}, t\right)\right| \frac{1}{\sqrt{t^{2}+2^{m j^{\prime}}}} d t
$$

where $\omega\left(\lambda, \lambda^{\prime}, t\right)=\bar{N}_{\lambda, \lambda^{\prime}}^{-1}$. But for every $\delta<\delta(m, \gamma)$ the Proposition 2.3 gives

$$
\left|\omega\left(\lambda, \lambda^{\prime}, t\right)\right| \leq C \omega_{\delta}\left(\lambda, \lambda^{\prime}\right),
$$

uniformly in $t$ (see the definition of $\omega_{\delta}\left(\lambda, \lambda^{\prime}\right)$ in Definition 2.1). Thus

$$
\left|M_{\lambda, \lambda^{\prime}}^{-1 / 2}\right| \leq C \omega_{\delta}\left(\lambda, \lambda^{\prime}\right) \int_{0}^{\infty} \frac{1}{\sqrt{t^{2}+2^{m j}} \sqrt{t^{2}+2^{m j^{\prime}}}} d t,
$$

hence

$$
\left|M_{\lambda, \lambda^{\prime}}^{-1 / 2}\right| \leq C \omega_{\delta}\left(\lambda, \lambda^{\prime}\right)\left(1+\left|j-j^{\prime}\right|\right) 2^{-m \sup \left\{j, j^{\prime}\right\} / 2},
$$

and since $\Phi_{\lambda}=\sum_{\lambda^{\prime}} M_{\lambda, \lambda^{\prime}}^{-1 / 2} \psi_{\lambda^{\prime}}$, we have $\Phi_{\lambda}=2^{-m j / 2} \sum_{\lambda^{\prime}} \theta_{\lambda, \lambda^{\prime}} \psi_{\lambda^{\prime}}$ where $\theta_{\lambda, \lambda^{\prime}}$ belongs to $\mathcal{M}^{\gamma^{\prime}}$ for any $\gamma^{\prime}<\delta(m, \gamma)$. Hence Proposition 2.2 .

We will now prove Proposition 2.3. In the following, $t \geq 0$ will be fixed; the dependancy of the coefficients in $t$ will often be forgotten, but all estimates will be uniform in $t$.

From Theorem 2.1 we have

$$
M=D N D,
$$

where $D$ is diagonal, $D=\operatorname{Diag}\left(2^{m j / 2}\right)$, and $N \in \mathcal{M}^{\gamma^{\prime}}$ for any $\gamma^{\prime}<$ $\delta(m, \gamma)$; then we get

$$
t^{2} \mathrm{Id}+M=D^{\prime} \bar{N} D^{\prime}
$$


with

$$
\bar{N}_{\lambda, \lambda^{\prime}}=\frac{t^{2} \delta_{\left(\lambda, \lambda^{\prime}\right)}+2^{m\left(j+j^{\prime}\right) / 2} N_{\lambda, \lambda^{\prime}}}{\sqrt{t^{2}+2^{m j}} \sqrt{t^{2}+2^{m j^{\prime}}}},
$$

where $\delta_{\left(\lambda, \lambda^{\prime}\right)}$ is the Kronecker symbol. As $N \in \mathcal{M}^{\gamma^{\prime}}$, for all $\gamma^{\prime}<\delta(m, \gamma)$ we obtain the same property for $\bar{N}$ and the first part of Proposition 2.3 is proved. We prove the second part after a study of invertibility of operators in the algebra $\mathcal{M}^{\gamma}$ performed in the next subsection. Basically, we will "freeze" the coefficients of the operator $t^{2} \operatorname{Id}+M$ at the center of the "numerical support" of the wavelets. The matrice of $t^{2} \mathrm{Id}+M$ in a wavelet basis will thus be approximated by another matrix that will be "invertible in $\mathcal{M}^{\gamma}$ for large $j$ 's". We will give a precise definition of these approximations of matrices, and this will lead to the "symbolic calculus" result stated in Theorem 2.1. In Subsection 2.4, these general results will be applied to the operator $t^{2} \mathrm{Id}+M$.

\subsection{The "quasi-ideals" $\mathcal{I}^{\gamma}$.}

Definition 2.3. A matrix $S$ belongs to $\mathcal{I}^{\delta}$ if $S \in \mathcal{M}^{\delta}$ and for all $\varepsilon>0$ exists $J$ such that $j \geq J$ or $j^{\prime} \geq J$ implies

$$
\left|S_{\lambda, \lambda^{\prime}}\right| \leq \varepsilon \omega_{\delta}\left(\lambda, \lambda^{\prime}\right)
$$

Remark. Suppose that $M \in \mathcal{M}^{\delta}$ and $\delta^{\prime}<\delta$. Then $M \in \mathcal{I}^{\delta^{\prime}}$ if for all $\varepsilon, C>0$, there exists $J$ such that if $j$ or $j^{\prime} \geq J$,

$$
\left|j-j^{\prime}\right| \leq C \text { and }\left|k 2^{-j}-k^{\prime} 2^{-j^{\prime}}\right| \leq C 2^{-j} \text { implies }\left|M_{\lambda, \lambda^{\prime}}\right| \leq \varepsilon .
$$

In fact if $j$ and $j^{\prime}$ are small, there is nothing to prove, and if either $\left|j-j^{\prime}\right|$ or $\left|k 2^{-j}-k^{\prime} 2^{-j^{\prime}}\right|$ is large, the result holds because $\omega_{\delta}\left(\lambda, \lambda^{\prime}\right) \leq$ $\varepsilon \omega_{\delta^{\prime}}\left(\lambda, \lambda^{\prime}\right)$.

Lemma 2.2. If $S \in \mathcal{I}^{\delta}$ and $M \in \mathcal{M}^{\delta}$ then for all $\delta^{\prime}<\delta, S M \in \mathcal{I}^{\delta^{\prime}}$ and $M S \in \mathcal{I}^{\delta^{\prime}}$.

Note that $\mathcal{I}^{\delta}$ is not an ideal in the algebra $\mathcal{M}^{\delta}$. The above lemma shows that it shares the same property as ideals if we are ready to admit an arbitrary small loss on the value of $\delta$.

Let

$$
\operatorname{dist}\left(\lambda, \lambda^{\prime}\right)=\left|j-j^{\prime}\right|+\left|\tilde{\lambda}-\tilde{\lambda}^{\prime}\right|
$$


which gives a distance on $\Lambda$. We can now sketch the proof of this lemma. We know that $S M \in \mathcal{M}^{\delta}$. If $\lambda$ and $\lambda^{\prime}$ are distant $\left(\left|j-j^{\prime}\right|\right.$ large or $\left|k / 2^{j}-k^{\prime} / 2^{j^{\prime}}\right|$ large) then $\omega_{\delta}\left(\lambda, \lambda^{\prime}\right) \leq \varepsilon \omega_{\delta^{\prime}}\left(\lambda, \lambda^{\prime}\right)$ hence the result in that case.

Suppose now that $\lambda$ and $\lambda^{\prime}$ are close; if $j$ is small, we have nothing to prove. If $j$ (and thus $j^{\prime}$ ) is large

$$
\begin{aligned}
\left|S M_{\lambda, \lambda^{\prime}}\right| & =\left|\sum_{\lambda^{\prime \prime}} S_{\lambda, \lambda^{\prime \prime}} M_{\lambda^{\prime \prime}, \lambda^{\prime}}\right| \\
& \leq \sum_{\lambda^{\prime \prime}} \varepsilon \omega_{\delta}\left(\lambda, \lambda^{\prime \prime}\right) \omega_{\delta}\left(\lambda^{\prime \prime}, \lambda^{\prime}\right) \\
& \leq C \varepsilon \omega_{\delta}\left(\lambda, \lambda^{\prime}\right) .
\end{aligned}
$$

Hence the lemma in this case. The proof for $M S$ is the same.

The importance of $\mathcal{I}^{\delta}$ comes from the following Proposition which shows that $\mathcal{I}^{\delta}$ will play a role similar to compact perturbations of invertible operators.

Proposition 2.4. Suppose that $M$ and $M^{-1}$ belong to $\mathcal{M}^{\delta}$ and that $S$ belongs to $\mathcal{I}^{\delta}$. If $M+S$ is invertible on $l^{2}$ then $(M+S)^{-1} \in \mathcal{M}^{\delta}$ and for all $\delta^{\prime}<\delta,(M+S)^{-1}-M^{-1} \in \mathcal{I}^{\delta^{\prime}}$.

Proof of Proposition 2.4. The first step is to reduce the proposition to the case where $S_{\lambda, \lambda^{\prime}}=0$ if $j \geq J$ or $j^{\prime} \geq J$. Let $\bar{S}$ the restriction of $Q$ to indexes $\left(\lambda, \lambda^{\prime}\right)$ such that $j \geq J$ or $j^{\prime} \geq J$. The norm of $\bar{S}$ in $\mathcal{I}^{\delta}$ can be made arbitrarily small by choosing $J$ large enough. The set of invertible elements in an algebra being open, $M+\bar{S}$ will be invertible if $J$ is large enough, hence the reduction that we claimed. We suppose now $S_{\lambda, \lambda^{\prime}}=0$ if $j \geq J$ or $j^{\prime} \geq J$. We have

$$
M+S=M\left(\operatorname{Id}+M^{-1} S\right)
$$

and

$$
\left(\mathrm{Id}-M^{-1} S\right)^{-1}=\mathrm{Id}+M^{-1} S\left(\operatorname{Id}-M^{-1} S\right)^{-1} .
$$

Let $E$ be the restriction of $M^{-1}$ to the indexes $\left(\lambda, \lambda^{\prime}\right)$ such that $j \leq J$ and $j^{\prime} \leq J$, and $E_{\lambda, \lambda^{\prime}}=0$ elsewhere. Then, one easily checks that $\operatorname{Id}-E S$ is invertible, and that $S\left(\operatorname{Id}-M^{-1} S\right)^{-1}=Q(\operatorname{Id}-E S)^{-1}$.

The fact that Id-ES belongs to $\mathcal{M}^{\delta}$ is equivalent to

$$
\left|(\operatorname{Id}-E S)_{\lambda, \lambda^{\prime}}\right| \leq \frac{C}{\left|1+\operatorname{dist}\left(\lambda, \lambda^{\prime}\right)\right|^{d+\delta}},
$$


for all $j, j^{\prime} \leq J$. The set of indexes we consider is a subset of $\mathbb{Z}^{d} \times$ $[0, \ldots, J]$. If it were a subset of $\mathbb{Z}$, a symbolic calculus result (see [21]) would show that (55) and the $l^{2}$-inversibility of (Id $\left.-E S\right)$ imply that estimate (55) holds for the invert of Id-ES, hence that (Id $\left.E S)^{-1}\right) \in \mathcal{M}^{\delta}$ since all other non diagonal entries of this matrix vanish. Actually, one checks by inspection that theorem of [21] also holds in the $d$-dimensional case.

Thus $(M+S)^{-1} \in \mathcal{M}^{\delta}$. Actually

$$
(M+S)^{-1}-M^{-1}=M^{-1} S(\mathrm{Id}-E S)^{-1} M^{-1}
$$

and since $S \in \mathcal{I}^{\delta}$, Lemma 2.2 implies that $(M+S)^{-1}-M^{-1} \in \mathcal{I}^{\delta^{\prime}}$ for all $\delta^{\prime}<\delta$.

Corollary 2.1. Suppose that $P \in \mathcal{M}^{\delta}$ and is selfadjoint positive and invertible on $l^{2}$. If there exists $Q \in \mathcal{M}^{\delta}$ such that $P Q-\operatorname{Id} \in \mathcal{I}^{\delta}$ then

$$
P^{-1} \in \mathcal{M}^{\delta} \quad \text { and } \quad P^{-1}-Q \in \mathcal{I}^{\delta^{\prime}}, \quad \text { for all } \delta^{\prime}<\delta .
$$

Proof. Let $J>0$ be given. By hypothesis $P Q=\mathrm{Id}+R+S$ where $R_{\lambda, \lambda^{\prime}}=0$ if $j \geq J$ or $j^{\prime} \geq J$ and $\varepsilon=\|S\|_{\mathcal{M}^{\gamma}}$ can be choosen arbitrarily small if $J$ is large enough. Let $I_{J}$ be the operator

$$
\begin{cases}\left(I_{J}\right)_{\lambda, \lambda^{\prime}}=1, & \text { if } \lambda=\lambda^{\prime} \text { and } j \leq J, \\ \left(I_{J}\right)_{\lambda, \lambda^{\prime}}=0, & \text { else. }\end{cases}
$$

For $\theta>0$ we consider $A\left(B+\theta I_{J}\right)=\mathrm{Id}+R+\theta P I_{J}+S$. First note that if $\theta$ is large enough $R+\operatorname{Id}+\theta A I^{J}$ is invertible on $l^{2}$ because, decomposing the matrices according to their action on $j \leq J$ and $j>J$, we can write

$$
P=\left(\begin{array}{ll}
P_{1} & P_{3} \\
P_{2} & P_{4}
\end{array}\right), \quad R=\left(\begin{array}{rr}
R^{\prime} & 0 \\
0 & 0
\end{array}\right)
$$

Thus

$$
\mathrm{Id}+R+\theta P I_{J}=\left(\begin{array}{cc}
R^{\prime}+\mathrm{Id}+\theta P_{1} & 0 \\
\theta P_{2} & \mathrm{Id}
\end{array}\right) .
$$

Since $P$ is selfadjoint positive invertible, $P_{1}$ has the same property, and

$$
\left\|P_{1}\right\| \leq\|P\| \quad \text { and } \quad\left\|P_{1}^{-1}\right\|\left\|P^{-1}\right\| .
$$


Thus choosing $\theta$ large enough, $\mathrm{Id}+R^{\prime}+\theta P_{1}$ is invertible, and, using the same argument as in the proof of Proposition 2.4, its inverse belongs to $\mathcal{M}^{\delta}$.

The inverse of $\operatorname{Id}+R+\theta P I^{J}$ is

$$
\left(\begin{array}{cc}
\left(\mathrm{Id}+R^{\prime}+\theta P_{1}\right)^{-1} & 0 \\
-\theta P_{2}\left(\mathrm{Id}+R^{\prime}+\theta P_{1}\right)^{-1} & \mathrm{Id}
\end{array}\right) .
$$

For a fixed $\theta$, the norms of Id $+R+\theta P I_{J}$ and of its inverse are bounded indenpendantly of $J$. Choosing $J$ large enough (which can be done independantly of the choice of $\theta), \varepsilon(=\|S\|)$ can be arbitrarily small, and thus Id $+R+\theta P I_{J}+S$ is invertible in $l^{2}$.

Applying Proposition 2.4, we see that $P\left(Q+\theta I_{J}\right)$ is invertible in $\mathcal{M}^{\delta}$. The same property holds for $P$ because $P^{-1}=\left(Q+\theta I_{J}\right)(P(Q+$ $\left.\left.\theta I_{J}\right)\right)^{-1}$.

Furthermore $P^{-1}-\left(Q+\theta I_{J}\right) \in \mathcal{I}^{\delta^{\prime}}$, for all $\delta^{\prime}<\delta$ and since $I_{J} \in \mathcal{I}^{\delta}$, we see that $P^{-1}-Q \in \mathcal{I}^{\delta^{\prime}}$, for all $\delta^{\prime}<\delta$.

\subsection{Application of the Quasi-ideals.}

We first end the proof of Proposition 2.3.

Recall that $\bar{N}$ is the matrix

$$
\bar{N}_{\lambda, \lambda^{\prime}}=\frac{\left(\left(t^{2} \mathrm{Id}+M\right) \psi_{\lambda} \mid \psi_{\lambda^{\prime}}\right)_{L^{2}}}{\theta_{\lambda} \theta_{\lambda^{\prime}}}
$$

and let

$$
\begin{aligned}
P_{\lambda, \lambda^{\prime}} & =\left(\theta_{\lambda} \theta_{\lambda^{\prime}}\right)^{-1}<\left(\left(t^{2}+\sigma(\lambda, \xi)\right) \hat{\psi}_{\lambda} \mid \hat{\psi}_{\lambda^{\prime}}\right)_{L^{2}}, \\
Q_{\lambda, \lambda^{\prime}} & =\theta_{\lambda} \theta_{\lambda^{\prime}}\left(\left(t^{2}+\sigma(\lambda, \xi)\right)^{-1} \hat{\psi}_{\lambda} \mid \hat{\psi}_{\lambda^{\prime}}\right)_{L^{2}} .
\end{aligned}
$$

Lemma 2.3. Under hypothesis $\mathrm{HA}(m, \gamma)$ the matrices $P$ and $Q$ belong to $\mathcal{M}^{\gamma^{\prime}}$ for $\gamma^{\prime}<\delta(m, \gamma)$.

Proof. As before we suppose $0 \leq j \leq j^{\prime}$. If $\left|j-j^{\prime}\right| \geq 2, P_{\lambda, \lambda^{\prime}}=0$ because of the supports of the $\hat{\psi}_{\lambda}$. If $\left|j-j^{\prime}\right|<2$ and $\left|\lambda-\lambda^{\prime}\right| \leq C 2^{-j}$,

$$
\left|P_{\lambda, \lambda^{\prime}}\right| \leq \int \frac{1+t^{2}+|\xi|^{m}}{1+t^{2}+2^{j m}} 2^{-d j / 2} 2^{-d j^{\prime} / 2}\left|\hat{\psi}\left(\frac{\xi}{2^{j}}\right)\right|\left|\hat{\psi}\left(\frac{\xi}{2^{j^{\prime}}}\right)\right| d \xi \leq C .
$$


If $\left|j-j^{\prime}\right|<2$ and $2^{j}\left|\lambda-\lambda^{\prime}\right|$ is large,

$$
P_{\lambda, \lambda^{\prime}}=\int \frac{t^{2}+\sigma(\tilde{\lambda}, \xi)}{\theta_{\lambda} \theta_{\lambda^{\prime}}} \hat{\psi}\left(\frac{\xi}{2^{j}}\right) \overline{\hat{\psi}}\left(\frac{\xi}{2^{j^{\prime}}}\right) e^{i\left(\lambda-\lambda^{\prime}\right) \xi} 2^{-d j / 2} 2^{-d j^{\prime} / 2} d \xi
$$

and integrating by parts in a chosen direction as above,

$$
\begin{aligned}
P_{\lambda, \lambda^{\prime}}= & \frac{1}{\left(\lambda_{l}-\lambda_{l}^{\prime}\right)^{r} 2^{\left(j+j^{\prime}\right) d / 2} \theta_{\lambda} \theta_{\lambda^{\prime}}} \\
& \cdot \sum_{p+q=r} \int \partial_{\xi_{l}}^{p} \sigma(\lambda, \xi) \partial_{\xi_{l}}^{q}\left(\psi\left(\frac{\xi}{2^{j}}\right) \overline{\hat{\psi}}\left(\frac{\xi}{2^{j^{\prime}}}\right)\right) d \xi,
\end{aligned}
$$

so that

$$
\left|P_{\lambda, \lambda^{\prime}}\right| \leq \frac{C}{\left|\lambda-\lambda^{\prime}\right|^{r} \theta_{\lambda} \theta_{\lambda^{\prime}}} \sum_{p+q=r} \int_{|\xi| \leq 2^{j} 8 \pi / 3}(1+|\xi|)^{m-p} 2^{-q j} d \xi
$$

and finally

$$
\left|P_{\lambda, \lambda^{\prime}}\right| \leq \frac{C}{\left(2^{j}\left|\lambda-\lambda^{\prime}\right|\right)^{r}}
$$

Hence Lemma 2.3 for $P$. The proof for $Q$ is similar.

Lemma 2.4. The matrix $\bar{N}-P$ belongs to $\mathcal{I}^{\gamma^{\prime}}$ and $P Q^{*}-$ Id belongs to $\mathcal{I}^{\gamma^{\prime}}$ for any $\gamma^{\prime}<\delta(m, \gamma)$.

Proof. By symmetry we can suppose $j \leq j^{\prime}$;

$$
(\bar{N}-P)_{\lambda, \lambda^{\prime}}=\frac{1}{\theta_{\lambda} \theta_{\lambda^{\prime}}} \int(\sigma(x, \xi)-\sigma(\lambda, \xi)) \hat{\psi}_{\lambda}(\xi) e^{i x \xi} \overline{\psi_{\lambda^{\prime}}(x)} d x d \xi
$$

Using the hypothesis HA $(m, \gamma)$,

$$
\begin{aligned}
&\left|(\bar{N}-P)_{\lambda, \lambda^{\prime}}\right| \leq \frac{C}{\theta_{\lambda} \theta_{\lambda^{\prime}}} \iint|x-\tilde{\lambda}|^{\varepsilon}(1+|\xi|)^{m+\varepsilon^{\prime}} 2^{-d j / 2} \\
& \cdot\left|\hat{\psi}\left(\xi 2^{-j}\right)\right| 2^{d j^{\prime} / 2}\left|\psi\left(2^{j^{\prime}} x-k^{\prime}\right)\right| d x d \xi \\
& \leq \frac{C}{\theta_{\lambda} \theta_{\lambda^{\prime}}} 2^{-\varepsilon j^{\prime}} 2^{d\left(j-j^{\prime}\right) / 2} \\
& \cdot \iint\left(1+2^{j}|\xi|\right)^{m+\varepsilon^{\prime}}|\hat{\psi}(\xi)| \\
& \cdot\left|x-2^{j^{\prime}}\left(\lambda-\lambda^{\prime}\right)\right| 2^{\varepsilon}|\psi(x)| d x d \xi
\end{aligned}
$$


and, because of (54),

$$
\left|(\bar{N}-P)_{\lambda, \lambda^{\prime}}\right| \leq \frac{C}{\theta_{\lambda} \theta_{\lambda^{\prime}}} 2^{-\varepsilon j^{\prime}} 2^{\varepsilon^{\prime} j}\left(1+2^{m j}\right) \leq C_{1} 2^{-\left(\varepsilon-\varepsilon^{\prime}\right) j} .
$$

Thus $\bar{N}-P \in \mathcal{I}^{\gamma^{\prime}}$.

Let us now prove the second result of Lemma 2.4. We have

$$
\begin{aligned}
\left(P Q^{*}\right)_{\lambda, \lambda^{\prime}}= & \sum_{\lambda^{\prime \prime}} \frac{\left(\left(t^{2}+\sigma(\lambda, \xi)\right) \hat{\psi}_{\lambda} \mid \hat{\psi}_{\lambda^{\prime \prime}}\right)_{L^{2}}}{\theta_{\lambda} \theta_{\lambda^{\prime \prime}}} \\
& \cdot \theta_{\lambda^{\prime \prime}} \theta_{\lambda^{\prime}}\left(\hat{\psi}_{\lambda^{\prime \prime}} \mid \frac{1}{t^{2}+\sigma\left(\lambda^{\prime}, \xi\right)} \hat{\psi}_{\lambda^{\prime}}\right)_{L^{2}} \\
= & \sum_{\lambda^{\prime \prime}} \frac{\theta_{\lambda^{\prime}}}{\theta_{\lambda}}\left(\left(t^{2}+\sigma(\lambda, \xi)\right) \hat{\psi}_{\lambda} \mid \hat{\psi}_{\lambda^{\prime \prime}}\right)_{L^{2}} \\
& \cdot\left(\hat{\psi}_{\lambda^{\prime \prime}} \mid \frac{1}{t^{2}+\sigma\left(\lambda^{\prime}, \xi\right)} \hat{\psi}_{\lambda^{\prime}}\right)_{L^{2}} \\
= & \frac{\theta_{\lambda^{\prime}}}{\theta_{\lambda}} \int \frac{t^{2}+\sigma(\lambda, \xi)}{t^{2}+\sigma\left(\lambda^{\prime}, \xi\right)} \hat{\psi}_{\lambda}(\xi) \overline{\hat{\psi}}_{\lambda^{\prime \prime}}(\xi) d \xi
\end{aligned}
$$

If $\left|j-j^{\prime}\right| \geq 2,\left(P Q^{*}\right)_{\lambda, \lambda^{\prime}}=0$ because the supports of $\hat{\psi}_{\lambda}$ and $\hat{\psi}_{\lambda^{\prime}}$ are disjoint. If $\lambda=\lambda^{\prime}$,

$$
\left(P Q^{*}\right)_{\lambda, \lambda^{\prime}}=\int \hat{\psi}_{\lambda}(\xi) \overline{\hat{\psi}}_{\lambda}(\xi) d \xi=1
$$

The remaining case is thus $\left|j-j^{\prime}\right|<2, \lambda \neq \lambda^{\prime}$. Since we can suppose that (54) holds, $\theta_{\lambda^{\prime}} / \theta_{\lambda}$ is of the order of magnitude of 1 , and we have to estimate

$$
\begin{aligned}
\chi_{\lambda, \lambda^{\prime}} & =\int \frac{t^{2}+\sigma(\lambda, \xi)}{t^{2}+\sigma\left(\lambda^{\prime}, \xi\right)} \hat{\psi}_{\lambda}(\xi) \overline{\hat{\psi}}_{\lambda^{\prime \prime}}(\xi) d \xi \\
& =\int \frac{\sigma(\lambda, \xi)-\sigma\left(\lambda^{\prime}, \xi\right)}{t^{2}+\sigma\left(\lambda^{\prime}, \xi\right)} \hat{\psi}_{\lambda}(\xi) \overline{\hat{\psi}}_{\lambda^{\prime \prime}}(\xi) d \xi
\end{aligned}
$$

(because of the orthogonality of the wavelets); but

$$
\begin{aligned}
\left|\chi_{\lambda, \lambda^{\prime}}\right| & \leq C \int \frac{\left|\lambda-\lambda^{\prime}\right|^{\varepsilon}(1+|\xi|)^{m+\varepsilon^{\prime}}}{(1+|\xi|)^{m}}\left|\hat{\psi}_{\lambda}\left(\frac{\xi}{2^{j}}\right)\right|\left|\hat{\psi}_{\lambda}\left(\frac{\xi}{2^{j^{\prime}}}\right)\right| d \xi \\
& \leq C\left|k-k^{\prime}\right|^{\varepsilon} 2^{j\left(\varepsilon^{\prime}-\varepsilon\right)} .
\end{aligned}
$$


This proves Lemma 2.4.

End of the Proof of Proposition 2.3. From Lemma 2.4, $\bar{N} Q^{*}-$ Id $\in \mathcal{I}^{\gamma^{\prime}}$, for all $\gamma^{\prime}<\delta(m, \gamma)$. From lemma $2.1, \bar{N}$ is invertible on $l^{2}$. Using Corollary 2.1, $\bar{N}^{-1} \in \mathcal{M}^{\gamma^{\prime}}$ and $\bar{N}^{-1}-Q^{*}$ belongs to $\mathcal{I}^{\gamma^{\prime}}$, for all $\gamma^{\prime}<\delta(m, \gamma)$, hence Proposition 2.3.

\subsection{Properties of the $\Phi_{\lambda}$.}

Let us check that Theorems 1.1, 1.2 and Proposition 1.2 are a direct consequence of the results given in the previous section. We first prove Theorem 1.1 which gives the localization and regularity of the wavelets $\Phi_{\lambda}$. Recall that

$$
\begin{gathered}
\Phi_{\lambda}=\sum_{\lambda^{\prime}} M_{\lambda, \lambda^{\prime}}^{-1 / 2} \psi_{\lambda^{\prime}}, \\
\left|M_{\lambda, \lambda^{\prime}}^{-1 / 2}\right| \leq C \omega_{\gamma}\left(\lambda, \lambda^{\prime}\right) 2^{-m \sup \left\{j, j^{\prime}\right\} / 2},
\end{gathered}
$$

thus

$$
\left|\partial^{s} \Phi_{\lambda}(x)\right| \leq C \sum_{\lambda^{\prime}} \omega_{\gamma}\left(\lambda, \lambda^{\prime}\right) 2^{-m \sup \left\{j, j^{\prime}\right\} / 2}\left|\partial^{s} \psi_{\lambda^{\prime}}(x)\right|
$$

where

$$
\partial^{s} \psi_{\lambda}(x)=2^{d j / 2}\left(\partial^{s} \psi\right)\left(2^{j} x-k\right) .
$$

If $|s|<m / 2$, we have

$$
\left|\partial^{s} \Phi_{\lambda}(x)\right| \leq C 2^{-(m / 2-|\alpha|) j} \sum_{\lambda^{\prime}} \tilde{\omega}_{\gamma}\left(\lambda, \lambda^{\prime}\right)\left|\partial^{s} \psi_{\lambda^{\prime}}(x)\right|
$$

Since $\partial^{s} \psi_{\lambda}$ are vaguelettes and $\omega_{\gamma} \in \mathcal{M}^{\gamma}$, using standard calculations explicited in [30], we deduce (18) and (19).

$$
\begin{aligned}
\text { If }|s|> & m / 2 \\
\left|\partial^{s} \phi_{\lambda}(x)\right| \leq & C 2^{-(m / 2-|s|) j} \\
& \cdot \sum_{\lambda^{\prime}} 2^{(m / 2-|s|) j} \omega_{\gamma}\left(\lambda, \lambda^{\prime}\right)\left(1+\left|j-j^{\prime}\right|\right) 2^{(m / 2-|s|) j^{\prime}} \partial^{s} \psi_{\lambda} .
\end{aligned}
$$

As the matrix $2^{(m / 2-|s|) j} \omega_{\gamma}\left(\lambda, \lambda^{\prime}\right)$ belongs to $\mathcal{M}^{\gamma-|s|-m / 2}(20)$ and (21) follow. 
As regards Theorem 1.2 we deduce from Lemma 2.4

$$
M_{\lambda, \lambda^{\prime}}^{-1 / 2}-\left\langle 2^{m j / 2} g_{\lambda} \mid \psi_{\lambda^{\prime}}\right\rangle=\alpha_{\lambda, \lambda^{\prime}}
$$

where the matrix $\left(2^{m \sup \left(j, j^{\prime}\right) / 2} \alpha_{\lambda, \lambda^{\prime}}\right)$ belongs to $\mathcal{I}^{\gamma^{\prime}}$ for all $\gamma^{\prime}<\delta(m, \gamma)$. The inequality of Theorem 1.2 is now straightforward.

As an application of the smoothness and decay properties of the wavelets, we now prove Proposition 1.2. We use the notation $\mathcal{A}\left(X_{A}, f\right)$ for the random variable associated to the function $f$ by the isomorphism $H_{A} \rightarrow \mathcal{H}$, see (1).

On account of Theorem 1.1 the results i), ii) can be proven exactly as in [3]. For the third result, we can use the following wavelet criterium (see [30]) for Besov spaces: if $\left(\psi_{\lambda}\right)_{\lambda \in \Lambda}$ is a wavelet basis of $L^{2}\left(\mathbb{R}^{d}\right)$, the function $f=\sum_{\lambda \in \Lambda} \alpha_{\lambda} \psi_{\lambda}$ belongs to the Besov space $B_{p, q}^{s}$ if and only if the sequence $\left\{2^{j(d(1 / 2-1 / p)+s)}\left(\sum_{j_{\lambda}=j}\left|\alpha_{\lambda}\right|^{p}\right)^{1 / p}\right\}_{j}$ belongs to $l^{q}$.

As the functions $2^{m j / 2} \Phi_{\lambda}$ define a Riesz basis of $L^{2}\left(\mathbb{R}^{d}\right)$, see Theorem 1.2, and satisfy wavelet localization properties, see Theorem 1.1, we have only to show that

$$
\sum_{j \geq J} 2^{j q(s+d / 2-d / p-m / 2)}\left(\sum_{\lambda \in U, j_{\lambda}=j}\left|\mathcal{A}\left(X_{A}, \Psi_{\lambda}\right)\right|^{p}\right)^{q / p}<\infty
$$

with probability one. The domain $U$ being bounded, the cardinal of $\left\{\lambda \in U, j_{\lambda}=j\right\}$ is of order $2^{j d}$ so that we get this inequality as consequence of the Borel-Cantelli Lemma when $s+d / 2-m / 2<0$.

\subsection{Equivalence in law of $X_{A}$ and $X_{A_{g}}$.}

Proof of Proposition 1.1. Let $g$ be the function defined in (13) and $A_{g}$ be the operator defined in (14). The symbol $\sigma_{g}$ of $A_{g}$ is given by

$$
\sigma_{g}(x, \xi)=g(\xi)+(1-g(\xi))^{2} \sigma(x, \xi)+r(x, \xi),
$$

with $r(x, \xi)$ a regularizing kernel. It is easy to check that $\sigma_{g}$ fulfills the conditions of HA $(m, \gamma)$. Moreover

$$
C_{1} \int|\xi|^{2 s}|\hat{f}(\xi)|^{2} d \xi \leq C_{1}(A(f) \mid f)_{L^{2}} \int\left(1+|\xi|^{2 s}\right)|\hat{f}(\xi)|^{2} d \xi
$$


hence the following equivalences

$$
\begin{aligned}
\left(A_{g}(f) \mid f\right)_{L^{2}} & \sim\|(\operatorname{Id}-G) f\|_{\dot{H}^{s}}^{2}+(G(f) \mid f)_{L^{2}} \\
& \sim \int(1-g(\xi))|\xi|^{2 s}|\hat{f}(\xi)|^{2} d \xi+\int g(\xi)|\hat{f}(\xi)|^{2} d \xi \\
& \sim \int(1+|\xi|)^{2 s}|\hat{f}(\xi)|^{2} d \xi .
\end{aligned}
$$

Using the notation $C(x, y)$ for the kernel of an operator $C$, we can write

$$
\begin{aligned}
X_{A_{g}}(x) & =\int_{\mathbb{R}^{d}} A_{g}^{-1 / 2}(x, y) d W(y), \\
X_{A}(x) & =\int_{\mathbb{R}^{d}} A^{-1 / 2}(x, y) d W(y),
\end{aligned}
$$

where $W(d y)$ denotes the brownian standard measure on $\mathbb{R}^{d}$.

In order to prove the equivalence of laws Law $\left(\left.X_{A_{g}}\right|_{U}\right)=$ Law $\left(\left.X_{A}\right|_{U}\right)$ for every bounded open subset $U$ of $\mathbb{R}^{d}$ we apply Theorem 8.6 of [32]. Therefore, we will check that

i) $C_{U}(x, y):=\left.A^{-1}\right|_{U \times U}(x, y)-\left.A_{g}^{-1}\right|_{U \times U}(x, y) \in H_{A_{g}}^{\otimes 2}(U \times U)$,

ii) -1 is not an eigenvalue of $C_{U}: H_{A_{g}} \rightarrow H_{A_{g}}$.

Let us consider the operator $B$ defined in (15). As the function $g$ belongs to $\mathcal{D}\left(\mathbb{R}^{d}\right)$ we know that $B$ is a regularizing operator

$$
A^{-1}-A_{g}^{-1}=A_{g}^{-1}\left(\left(I+B A_{g}^{-1}\right)^{-1}-I\right)
$$

and

$$
\left(\left(I+B A_{g}^{-1}\right)^{-1}=\sum_{n \geq 0}(-1)^{n}\left(B A_{g}^{-1}\right)^{n} .\right.
$$

Now if we consider the restrictions to open bounded $U$ which are small enough, the last serie converges and the operator $A^{-1}-A_{g}^{-1}$ is of Hilbert-Schmidt type with a spectral radius less than 1 , so that condition ii) is satisfied.

For the first condition, it is sufficient to show that

$$
(-\Delta)_{x}^{m / 4}(-\Delta)_{y}^{m / 4} C(x, y) \in L_{\mathrm{loc}}^{2}\left(\mathbb{R}^{d} \otimes \mathbb{R}^{d}\right) .
$$

But, as before

$$
\begin{aligned}
(-\Delta)_{x}^{m / 4}(-\Delta)_{y}^{m / 4} C(x, y) & \\
& =\sum_{n \geq 1}(-1)^{n}(-\Delta)_{x}^{m / 4} A_{g}^{-1}\left(B A_{g}^{-1}\right)^{n}(-\Delta)_{x}^{m / 4}(x, y)
\end{aligned}
$$


which converges in $L^{2}(U \times U)$ for $U$ small enough, since $A_{g}^{-1}$ is an operator of order $-m$ and $B$ is regularizing.

Finally we obtain the equivalence of laws for every bounded open subset $U$ of $\mathbb{R}^{d}$, by decomposing $U$ in a finite number of small enough open subsets.

\subsection{Quadratic variations.}

In this paragraph, we will prove Lemma 1.2. For this purpose, we will study some quadratic variations related to wavelets.

For $y \in \mathbb{R}^{d}$ and $s \in \mathbb{N}^{d},|s|=l$, we define

$$
c_{1, s}^{2}(y)=\limsup _{x \rightarrow y} \frac{1}{|x-y|^{\alpha} \delta_{\alpha}(|x-y|)} \sqrt{\sum_{\lambda}\left|\partial^{s} \Phi_{\lambda}(x)-\partial^{s} \Phi_{\lambda}(y)\right|^{2}},
$$

where $\delta_{\alpha}(h)=1$ if $\alpha<1$ and $\delta_{\alpha}(h)=\sqrt{\log (1 / h)}$ if $\alpha=1$. If $n$ is the integer defined by $2^{-n} \leq|x-y|<2^{1-n}$ we deduce from (18)

a) for $j \leq n$,

$$
\sum_{k, l}\left|\partial^{s} \Phi_{\lambda}(x)-\partial^{s} \Phi_{\lambda}(y)\right|^{2} \leq C|x-y|^{2} 2^{2(1-\alpha) j}
$$

b) for $j>n$,

$$
\sum_{k, l}\left|\partial^{s} \Phi_{\lambda}(x)-\partial^{s} \Phi_{\lambda}(y)\right|^{2} \leq C 2^{-2 j \alpha}
$$

Summing up these inequalities for $j \geq 0$ yields

$$
\sum_{\lambda}\left|\partial^{s} \Phi_{\lambda}(x)-\partial^{s} \Phi_{\lambda}(y)\right|^{2} \leq C\left(u_{\alpha, n}|x-y|^{2}+2^{-2 n \alpha}\right),
$$

with $u_{\alpha, n}=n$ (respectively $2^{2(1-\alpha) n}$ ) if $\alpha=1$ (respectively $<1$ ). As $2^{-n} \leq|x-y|<2^{1-n}$,

$$
c_{1, s}^{2}(y) \leq C<\infty, \quad \text { for all } y \in \mathbb{R}^{d} .
$$

Let us now show that $c_{1, s}^{2}$ is Hölderian of order $\varepsilon^{\prime \prime}:=\varepsilon-\varepsilon^{\prime}$, where $\varepsilon$ and $\varepsilon^{\prime}$ are defined in (7). Let us distinguish two cases. 
Case 1. $\alpha<1$.

For $\varepsilon>0$ fixed, using as above the results of Theorem (1.1), we have

$$
\begin{gathered}
\sum_{j \leq n-\log _{2}(1 / \varepsilon)} \frac{\left|\partial^{s} \Phi_{\lambda}(x)-\partial^{s} \Phi_{\lambda}(y)\right|^{2}}{|x-y|^{2 \alpha}} \leq C \varepsilon^{2(1-\alpha)}, \\
\sum_{j \geq n+\log _{2}(1 / \varepsilon)} \frac{\left|\partial^{s} \Phi_{\lambda}(x)-\partial^{s} \Phi_{\lambda}(y)\right|^{2}}{|x-y|^{2 \alpha}} \leq C \varepsilon^{2 \alpha}, \\
\sum_{\lambda \in \Lambda_{y, n, \varepsilon}} \frac{\left|\partial^{s} \Phi_{\lambda}(x)-\partial^{s} \Phi_{\lambda}(y)\right|^{2}}{|x-y|^{2 \alpha}} \leq C \varepsilon^{2(1-\alpha)},
\end{gathered}
$$

where in the last inequality

$$
\Lambda_{y, n, \varepsilon}=\left\{\lambda \in \Lambda: n-\log _{2}\left(\frac{1}{\varepsilon}\right)<j<n+\log _{2}\left(\frac{1}{\varepsilon}\right),|y-\lambda|>\frac{2^{-n}}{\varepsilon}\right\} .
$$

Let $\varepsilon=1 / n$; when $n$ grows to $\infty$ the value of $c_{1, s}(y)$ is given by the sum restricted to $V_{y, n, \varepsilon}=\Lambda \backslash \Lambda_{y, n, \varepsilon}$. Define $h_{\lambda}$ by its Fourier transform

$$
\hat{h}_{\lambda}(\xi)=\frac{1}{\sqrt{\sigma(y, \xi)}} \hat{\psi}_{\lambda}(\xi)
$$

and observe that the estimates (18), (19) hold for $h_{\lambda}$. Then inequalities (56), (57) and (58) hold with $\Phi_{\lambda}$ replaced by $h_{\lambda}$. Using Theorem 1.2, for $n$ large enough

$$
\begin{aligned}
\sum_{\lambda \in V_{y, n, \varepsilon}}|| \partial^{s} \Phi_{\lambda}(x)-\left.\partial^{s} \Phi_{\lambda}(y)\right|^{2}-\mid \partial^{s} g_{\lambda}(x)- & \left.\partial^{s} g_{\lambda}(y)\right|^{2} \mid \\
& \leq C \varepsilon^{\prime}|x-y|^{2 \alpha}
\end{aligned}
$$

thus hypothesis HA $(m, \gamma)$ implies that for $\lambda \in V_{y, n, \varepsilon}(59)$ holds for $h_{\lambda}$ instead of $g_{\lambda}$. Thus

$$
c_{1, s}^{2}(y)=\limsup _{x \rightarrow y} \sum_{\lambda} \frac{\left|\partial^{s} h_{\lambda}(x)-\partial^{s} h_{\lambda}(y)\right|^{2}}{|x-y|^{2 \alpha}} .
$$

Define the function $H$ by

$$
\hat{H}(\xi)=\frac{1}{\sqrt{\sigma(y, \xi)}},
$$


so that

$$
\left|\partial^{s} h_{\lambda}(x)-\partial^{s} h_{\lambda}(y)\right|^{2}=\left(\partial^{s} H(x-\cdot)-\partial^{s} H(y-\cdot) \mid \psi_{\lambda}\right)_{L^{2}}^{2} .
$$

Since $\psi_{\lambda}$ is an orthonormal basis of $L^{2}$,

$$
\begin{aligned}
c_{1, s}^{2}(y) & =\limsup _{x \rightarrow y} \frac{1}{|x-y|^{2 \alpha}} \int\left|\left(e^{i x \xi}-e^{i y \xi}\right) \frac{(i \xi)^{s}}{\sqrt{\sigma(y, \xi)}}\right|^{2} d \xi \\
& =\limsup _{u \rightarrow 0} \frac{4}{|u|^{2 \alpha}} \int \sin ^{2}\left(\frac{u \xi}{2}\right) \frac{|\xi|^{2|s|}}{\sigma(y, \xi)} d \xi .
\end{aligned}
$$

We want to bound $I(z, u)-I(y, u)$ where

$$
I(y, u):=\frac{1}{|u|^{2 \alpha}} \int \sin ^{2}\left(\frac{u \xi}{2}\right) \frac{|\xi|^{2|s|}}{\sigma(y, \xi)} d \xi .
$$

Recalling that $|s|=l, 2(l+\alpha)=m-d$, and using the change of variable $\zeta=|u| \xi$,

$$
\begin{aligned}
|I(z, u)-I(y, u)| \leq \int & \frac{|\zeta /| u||^{m}}{|\sigma(y, \zeta /|u|) \sigma(z, \zeta /|u|)|} \\
& \cdot|\sigma(y, \zeta /|u|)-\sigma(z, \zeta /|u|)| \frac{\sin ^{2}(\zeta u / 2|u|)}{|\zeta|^{d+2 \alpha}} d \zeta .
\end{aligned}
$$

As

$$
\begin{aligned}
|\sigma(\cdot, \xi)| & \geq C_{1}(1+|\xi|)^{m}, \\
|\sigma(y, \xi)-\sigma(z, \xi)| & \leq C_{2}(1+|\xi|)^{m}|y-z|^{\varepsilon^{\prime \prime}},
\end{aligned}
$$

we get, using $0 \leq \sin ^{2}(t) \leq \min \left\{1, t^{2}\right\}$,

$$
\begin{aligned}
|I(z, u)-I(y, u)| & \leq C_{3}|y-z|^{\varepsilon^{\prime \prime}}\left(\int_{0}^{1} r^{-2 \alpha+1} d r+\int_{1}^{\infty} r^{-1-2 \alpha} d r\right) \\
& \leq C_{4}|y-z|^{\varepsilon^{\prime \prime}}
\end{aligned}
$$

and thus the $\varepsilon^{\prime \prime}$-Hölder property for $c_{1, s}^{2}$.

Case 2. $\alpha=1$.

The difference with the previous case is that

$$
\sum_{k, l} \frac{\left|\partial^{s} \Phi_{\lambda}(x)-\partial^{s} \Phi_{\lambda}(y)\right|^{2}}{|x-y|^{2 \alpha}}
$$


no longer decreases (as $j$ increases). We must replace the set $\Lambda_{y, n, \varepsilon}$ by

$$
\tilde{\Lambda}_{y, n, \varepsilon}=\left\{\lambda \in \Lambda: \sqrt{n} \leq j<n+\log _{2}\left(\frac{1}{\varepsilon}\right),|y-\lambda|>\frac{2^{-n}}{\varepsilon}\right\}
$$

and define now $V_{y, n, \varepsilon}=\Lambda \backslash \tilde{\Lambda}_{y, n, \varepsilon}$. We can then proceed exactly as above and obtain

$$
c_{1, s}^{2}(y)=\limsup _{x \rightarrow y} \sum_{\lambda} \frac{\left|\partial^{s} h_{\lambda}(x)-\partial^{s} h_{\lambda}(y)\right|^{2}}{|x-y|^{2} \log \left(\frac{1}{|x-y|}\right)}=4 \tilde{I}(y, u)
$$

with

$$
\tilde{I}(y, u)=\frac{1}{|u|^{2} \log (1 /|u|)} \int \sin ^{2}\left(\frac{u \xi}{2}\right) \frac{|\xi|^{2 l}}{\sigma(y, \xi)} d \xi
$$

Using again (61), (62), we see that $c_{1, s}^{2}$ is Hölder of order $\varepsilon^{\prime \prime}$. Hence Lemma 1.2. Let us, still in the case $\alpha=1$, consider the expression

$$
c_{2, s}^{2}(y)=\limsup _{x \rightarrow y} \frac{1}{|x-y|} \sqrt{\sum_{\lambda}\left|\partial^{s} \Phi_{\lambda}(x)-2 \partial^{s} \Phi_{\lambda}\left(\frac{x+y}{2}\right)+\partial^{s} \Phi_{\lambda}(y)\right|^{2}}
$$

with $y \in \mathbb{R}^{d}, s \in \mathbb{N}^{d},|s|=l$. Using once again the bounds for $\partial^{r} \Phi_{\lambda}$ (with $|r|=l+2$ ) given in (18), we have for $n:=\left[\log _{2}(|x-y|)\right]$

$$
\sum_{k, l}\left|\partial^{s} \Phi_{\lambda}(x)-2 \partial^{s} \Phi_{\lambda}\left(\frac{x+y}{2}\right)+\partial^{s} \Phi_{\lambda}(y)\right|^{2} \leq C|x-y|^{4} 2^{2 j},
$$

if $j \leq n$, and

$$
\sum_{k, l}\left|\partial^{s} \Phi_{\lambda}(x)-2 \partial^{s} \Phi_{\lambda}\left(\frac{x+y}{2}\right)+\partial^{s} \Phi_{\lambda}(y)\right|^{2} \leq C 2^{-2 j}
$$

if $j>n$. Thus, after summation

$$
c_{2, s}^{2}(y) \leq C<\infty, \quad \text { for all } y \in \mathbb{R}^{d} .
$$

The required smoothness of $c_{2, s}$ follows as above. 


\section{Sums on the $2^{d}$-adic tree.}

The key idea to prove the law of uniform modulus in the critical case $\alpha=1$ is to notice the relationship between the expression of the process $X_{A}$ decomposed on the $\Phi_{\lambda}$ 's and sums of normal random variable on the $2^{d}$-adic tree.

As explained in Section 1, we have to study when $n=\log _{2}(\mid x-$ $y \mid) \rightarrow \infty$, the following sums

$$
\frac{\left|\partial^{s} X_{A}(x)-\partial^{s} X_{A}(y)\right|}{|x-y|} \simeq \text { Const. } \sum_{\lambda \in \Lambda \cap D, j_{\lambda} \leq n} \mathbf{1}_{c_{\lambda}(x)} \xi_{\lambda},
$$

see (35). But the last sum is exactly the sum of Gaussian standard random variables on the paths of length $n$ of a $2^{d}$-adic tree. This will be performed after introducing some notations.

Let $\mathcal{T}$ be the $2^{d}$-adic tree of root $*$ (each "father" has $2^{d}$ children). We denote by $\bar{L}$ the set $\{0,1\}^{d}$ and by $L$ the set $\bar{L} \backslash\{(0, \ldots, 0)\}$. The elements of $\mathcal{T}$ can be coded in the following manner

$$
t=t_{0} t_{1} t_{2} \cdots t_{j}, \quad \text { with } j \in \mathbb{N}, t_{0}=*, t_{i} \in \bar{L} \text { for } i=1, \ldots, j .
$$

The lenght $j$ of $t$ is denoted by $|t|:=j$. For integers $0 \leq k \leq|t|$ we write

$$
\underline{t}_{k}=t_{0} t_{1} \cdots t_{k}
$$

so that the path from the root to $t$ is

$$
C_{t}^{*}=\left\{*, \ldots, \underline{t}_{k}, \ldots, t\right\}
$$

Let $(\Omega, \mathcal{F}, \mathbb{P})$ a probability space on which is defined the i.i.d. family $\left\{\xi_{t}, t \in \mathcal{T}\right\}$ of Gaussian standard random variables. We set

$$
\begin{aligned}
& S(t)=\sum_{s \in C_{t}^{*}} \xi_{s}, \\
& S_{n}^{*}=\max _{|t|=n} S(t) .
\end{aligned}
$$

Proposition 3.1. With the above notations the following limit holds

$$
\limsup _{n \rightarrow \infty} \frac{S_{n}^{*}}{n}=\sqrt{2 d \log 2}
$$


$\mathbb{P}$ almost surely.

The aim of this paragraph is to prove Proposition 3.1 and to give a corollary used in the proof of Theorem 1.3 in the critical case $(\alpha=1)$. A proof of Proposition 3.1 can be found in a recent work, see [10], where more general trees are considered. Our proof is very different and intends to show the production of asymptotic independent sums in the tree, so that we the study will be reduced to the i.i.d. case.

A few more notations will be needed.

The sub-tree of index $j$ of $\mathcal{T}$ is defined by

$$
\mathcal{T}_{j}=\{t \in \mathcal{T}:|t| \leq j\} .
$$

The set of leaves of $\mathcal{T}_{j}$ is defined by

$$
\wedge_{j}=\{t \in \mathcal{T}:|t|=j\} .
$$

The tree is ordered by $u \succ t$ which means that $t \in C_{u}^{*}$. The cells $\left(q_{j}(t), t \in \mathcal{T}_{j}\right)$ are defined by

$$
q_{j}(t)=\left\{s \in \wedge_{j}: s \succ t\right\} .
$$

We now define the Haar basis of $l^{2}\left(\wedge_{j}\right)$.

For $l \in L$ and $\theta \in \bar{L}$, let

$$
\varepsilon_{l}(\theta)=\prod_{i=1}^{d} \varepsilon_{l_{i}}\left(\theta_{i}\right),
$$

where $\varepsilon_{0}(0)=\varepsilon_{0}(1)=\varepsilon_{1}(0)=+1, \varepsilon_{1}(1)=-1$.

Let us now define the functions $\psi_{j, s}^{l}$ on $l^{2}\left(\wedge_{j}\right)$ by

$$
\psi_{j, s}^{l}(t)=2^{d(|s|-j) / 2} \mathbf{1}_{\left\{t \in q_{j}(s): t \neq s\right\}} \varepsilon_{l}\left(t_{|s|+1}\right) .
$$

If we add to the family $\left\{\psi_{j, s}^{l}\right\}_{s \in \delta_{j-1}, l \in L}$ the function $\psi_{\phi}$ which is identically $2^{d j}$ on $l^{2}\left(\wedge_{j}\right)$ and if we set $|\phi|=-1$, we obtain the following result whose proof is straightforward.

Lemma 3.1. The family $\left\{\psi_{j, t}^{l}\right\}_{-1 \leq t \leq j-1, l \in L}$ is an orthonormal basis of $l^{2}\left(\wedge_{j}\right)$. 


\subsection{Upper bound.}

For $t, u \in \mathcal{T}$ let

$$
\begin{gathered}
t u=* t_{1} \cdots t_{|t|} u_{1} \cdots u_{|u|} \\
S_{t}(u)=S(t u)-S(t), \\
\left(S_{t}\right)_{j}^{*}=\max _{|u|=j} S_{t}(u)
\end{gathered}
$$

so that

$$
S_{j}^{*}=\xi_{*}+\max _{|t|=1}\left\{S_{t}\right\}_{j-1}^{*} \cdot
$$

If $\mathbb{E}_{\xi_{*}}$ denotes the expectation with respect to the law of $\xi_{*}$, we get

$$
\begin{aligned}
\mathbb{P}\left(S_{j}^{*}<\theta\right) & =\mathbb{E}_{\xi_{*}}\left(\prod_{|t|=1}^{d} \mathbb{P}\left(\xi_{*}+\left(S_{t}\right)_{j-1}^{*}<\theta\right)\right) \\
& =\mathbb{E}_{\xi_{*}}\left(\mathbb{P}\left(\xi_{*}+S_{j-1}^{*}<\theta\right)^{2^{d}}\right) \\
& \geq \mathbb{P}\left(\xi_{*}+S_{j-1}^{*}<\theta\right)^{2^{d}} \quad \text { (Jensen) }
\end{aligned}
$$

and by induction on $j$,

$$
\mathbb{P}\left(S_{j}^{*}<\theta\right) \geq \mathbb{P}\left(\xi_{*}+\cdots+\xi_{j}<\theta\right)^{2^{j d}} .
$$

Let $\eta$ be a gaussian normal random variable and $\theta=\beta(j+1)$;

$$
\begin{aligned}
\mathbb{P}\left(S_{j}^{*}>\beta(j+1)\right) & \leq 1-(1-\mathbb{P}(\eta>\beta \sqrt{j+1}))^{2^{j d}} \\
& \leq 2^{j d} \mathbb{P}(\eta>\beta \sqrt{j+1}) \\
& \leq \frac{2^{j d} e^{-\beta^{2}(j+1)}}{\sqrt{2+\beta(j+1)}}
\end{aligned}
$$

using a classical estimation on the gaussian tail.

Choosing $\beta>\sqrt{2 d \log 2}$, we have

$$
\sum_{j} \mathbb{P}\left(S_{j}^{*}>\beta(n+1)\right)<\infty,
$$

and from the Borel-Cantelli Lemma we can conclude

$$
\limsup _{j \rightarrow \infty} \frac{S_{j}^{*}}{j} \leq \sqrt{2 d \log 2}
$$


$\mathbb{P}$ almost surely.

\subsection{Lower bound.}

Let $G_{j}(t, u)=\mathbb{E}[S(t) S(u)]$ be the covariance of $S$ on $\wedge_{j}$. In the following lemma we give the spectral decomposition of $G_{j}$. We define

$$
\alpha_{s}=\frac{2^{d(j-|s|)}-1}{2^{d}-1} .
$$

Lemma 3.2. For $t, u \in \wedge_{j}$ we have

$$
G_{j}(t, u)=\sum_{\substack{-1 \leq|s| \leq j-1 \\ l \in L}} \alpha_{s} \psi_{j, s}^{l}(t) \psi_{j, s}^{l}(u) .
$$

This lemma is a direct consequence of the obvious formula

$$
G_{j}(t, u)=\sum_{k=1}^{j} \delta_{\underline{t}_{k}, \underline{u}_{k}},
$$

where $\underline{t}_{k}=* t_{1} t_{2} \cdots t_{k}$, and $\delta$ is the Kroneker symbol.

Now we define the kernel $G_{j}^{-1 / 2}(t, u)$ by

$$
G_{j}^{-1 / 2}(t, u)=\sum_{\substack{-1 \leq|s| \leq j-1 \\ l \in L}} \alpha_{s}^{-1 / 2} \psi_{j, s}^{l}(t) \psi_{j, s}^{l}(u)
$$

and the random variables $\eta_{s}$ by

$$
\eta(s):=\sum_{t \in \wedge_{j}} G_{j}^{-1 / 2}(s, t) S(t), \quad s \in \wedge_{j}
$$

Lemma 3.3. The family $\eta(s), s \in \wedge_{j}$ is i.i.d. with common law $\mathcal{N}(0,1)$.

The proof is immediate since in the gaussian centered case $\mathbb{E}(\xi \eta)=$ 0 is equivalent to the independance of $\xi$ and $\eta$. 
Let us introduce some more notations. For $x>0$ let $l(x)=$ $[\log (x) / d \log 2]=\left[\log _{2^{d}}(x)\right], \tilde{j}=j-l(j)$ and $\tilde{\eta}_{j}(s)=\sum_{t \in q_{j}(s)} \eta(t) / j$, the last expression being the arithmetic mean on $q_{j}(s)$ when $|s|=\tilde{j}$.

The upper bound will be obtained by proving

(66) $\quad \limsup _{j \rightarrow \infty} \frac{1}{j} \max _{|s|=\tilde{j}} S(s) \geq \sqrt{2 d \log 2}-\varepsilon, \quad$ for all $\varepsilon>0$.

First, we observe that

$$
\tilde{\eta}_{j}(s)=\frac{1}{j} \sum_{t \in q_{j}(s)} \sum_{\substack{u \in \wedge_{j} \\|r| \leq j-1}} \alpha_{\substack{\mid \\ l \in L}}^{-1 / 2} \psi_{j, r}^{l}(t) \psi_{j, r}^{l}(u) S(u) .
$$

As $\sum_{t \in q_{j}(s)} \psi_{j, r}^{l}(t)$ is equal to $j \varepsilon_{l}\left(\underline{s}_{|r|+1}\right) 2^{d(|r|-j) / 2}$ or to 0 according to $(r \prec s, r \neq s)$ or not, the expression (67) can be simplified in

$$
\tilde{\eta}_{j}(s)=\sum_{u \in \wedge_{j}} \sum_{\substack{r \prec s \\ l \in L}} \varepsilon_{l}\left(\underline{s}_{|r|+1}\right) \alpha_{r}^{-1 / 2} 2^{d(|r|-j) / 2} \psi_{j, r}^{l}(u) S(u) .
$$

We consider now the decomposition $\tilde{\eta}_{j}(s)=\eta_{j}^{\varepsilon}(s)+\eta_{j}^{0}(s)$ with

$$
\begin{gathered}
\eta_{j}^{\varepsilon}(s)=\sum_{u \in q_{j}\left(\underline{s}_{j(s)}\right)} \sum_{\substack{l \in L \\
r \prec s}} \alpha_{r}^{-1 / 2} 2^{d(|r|-j) / 2} \psi_{j, r}^{l}(u) S(u), \\
\eta_{j}^{0}(s)=\sum_{u \in \wedge_{j} \backslash q_{j}\left(\underline{s}_{j(s)}\right)} \sum_{\substack{l \in L \\
r \prec s}} \varepsilon_{l}\left(\underline{s}_{|r|+1}\right) \alpha_{r}^{-1 / 2} 2^{d(|r|-j) / 2} \psi_{j, r}^{l}(u) S(u)
\end{gathered}
$$

and $j(\varepsilon)=j-2 l(j / \varepsilon)$. Using the same cancellation property as above, the summation in $\eta_{j}^{0}$ can be restricted to $r \prec \underline{s}_{j(\varepsilon)}$. The following Lemma allows us to bound $\left|\eta_{j}^{0}\right|$.

Lemma 3.4. For every $\varepsilon>0$ there exists a random variable $N$ and $a$ constant $C$ such that for all $s \in \wedge_{j(\varepsilon)}$

$$
\sum_{u \in \wedge_{j}} \sum_{\substack{l \in L \\ r \prec s}}\left|\alpha_{r}^{-1 / 2} 2^{d(|r|-j) / 2} \psi_{j, r}^{l}(u) S(u)\right| \leq C \varepsilon, \quad \text { on }\{j \geq N\},
$$

$\mathbb{P}$ almost surely. 
Proof. Let $\theta>\sqrt{2 d \log 2}$, and let $N$ be some random variable such that $\left|S_{j}^{*}\right| \leq \theta j$ on $\{N(\omega)<j\}$ which we determined during proof of the upper bound. From the inequalities $\left|\alpha_{r}^{-1 / 2} \psi_{j, r}^{l}(u)\right| \leq 2^{d(|r|-j)}$ and card $\left\{u \in \wedge_{j}: u \succ r\right\} \leq 2^{d(j-|r|)}$, we get

$$
\sum_{u \in \wedge_{j}} \sum_{\substack{l \in L \\ r \prec s}}\left|\alpha_{r}^{-1 / 2} 2^{d(|r|-j) / 2} \psi_{j, r}^{l}(u) S(u)\right| \leq \theta j \sum_{k=0}^{j(\varepsilon)} 2^{k-d / 2}=C \varepsilon,
$$

on $\{j>N(\omega)\}$. Hence Lemma 3.4.

Consider now the following decomposition

$$
\eta_{j}^{\varepsilon}(s)=\sum_{\substack{v \succ \underline{s}_{j(\varepsilon)} \\|v|=j-2 l(j)}} B_{v}+C_{v}
$$

where

$$
\begin{aligned}
B_{v} & =\left(\sum_{u \in q_{j}(v)} \sum_{l, r \prec s} \mu_{u, l, r}\right) S(v), \\
C_{v} & =\sum_{u \in q_{j}(v)} \sum_{l, r \prec s} \mu_{u, l, r} S_{v}(u),
\end{aligned}
$$

and

$$
\mu_{u, l, r}=\alpha_{r}^{-1 / 2} \varepsilon_{l}\left(\underline{s}_{|r|+1}\right) 2^{d(|r|-j) / 2} \psi_{j, r}^{l}(u) .
$$

Lemma 3.5.

$$
\lim _{j \rightarrow \infty} \sum_{\substack{v \succ \underline{s}_{j}(\varepsilon) \\ v \mid=j-2 l(j) \\ v \neq \underline{s}_{j-2 l(j)}}} B_{v}=0
$$

$\mathbb{P}$ almost surely.

Proof. The summation on $r$ is in this case reduced to $r \prec \underline{s}_{(j-2 l(j))}$. We have

$$
\sum_{\substack{r \prec \underline{s}_{j-2 l(j)} \\ l}}\left|\mu_{u, l, r}\right| \leq \sum_{k=0}^{j-2 l(j)} 2^{3(k-j) d / 2} \leq j^{-3}
$$


and thus

$$
\left|\sum_{u \in q_{j}(v)} \sum_{\substack{l \\ r \prec \underline{s}_{j-2 l(j)}}} \mu_{u, l, r}\right| \leq j^{-1} .
$$

As we can bound the cardinal of $\left\{v \succ \underline{s}_{j(\varepsilon)},|v|=j-2 l(j)\right\}$ by $2^{2 d l(1 / \varepsilon)}$, we get when $j \rightarrow \infty$

$$
A:=\sum_{\substack{v \succ \underline{s}_{j(\varepsilon)} \\|v|=j-2 l(j) \\ v \neq \underline{s}_{j-2 l(j)}}} \sum_{\substack{u \in q_{j}(v) \\ r \prec \underline{s}_{j-2 l(j)} \\ l}} \mu_{u, l, r} S\left(\underline{s}_{j(\varepsilon)}\right) \rightarrow 0,
$$

$\mathbb{P}$ almost surely. We still have to study

$$
R:=\left(\sum_{\substack{v \succ \underline{s}_{j(\varepsilon)} \\|v|=j-2 l(j) \\ v \neq \underline{s}_{j}-2 l(j)}} B_{v}\right)-A .
$$

But

$$
R=\left(\sum_{\substack{u \in q_{j}(v) \\ r \prec \underline{s}_{j-2 l(j)} \\ l}} \mu_{u, l, r}\right)\left(\sum_{\substack{v \succ \underline{s}_{j(\varepsilon)} \\|v|=j-2 l(j) \\ v \neq \underline{s}_{j-2 l(j)}}} S_{\underline{s}_{j(\varepsilon)}}(v)\right)
$$

and using the independance of the random variable $S_{\underline{s}_{j(\varepsilon)}}(v)$ we have $\mathbb{E}\left(R^{2}\right) \leq C j^{-2}$. The convergence we claimed is now clear.

Lemma 3.6. The following limit holds

$$
\lim _{j \rightarrow \infty} \sum_{\substack{v \succ \underline{s}_{j(\varepsilon)} \\|v|=j-2 l(j)}} C_{v}=0,
$$

$\mathbb{P}$ almost surely.

Proof. Using the definition of $C_{v}$ we can write

$$
\sum_{\substack{v \succ \underline{s}_{j(\varepsilon)} \\|v|=j-2 l(j) \\ v \neq \underline{s}_{j-2 l(j)}}} C_{v}=Q_{j}+R_{j}
$$


where

$$
R_{j}=\sum_{u \in q_{j}\left(\underline{s}_{j-2 l(j)}\right)} \sum_{\underline{s}_{j-2 l(j)} \prec r \prec s} \mu_{u, l, r} S_{\underline{s}_{j-2 l(j)}}(u),
$$

so that only $r \prec \underline{s}_{j-2 l(j)}$ are involved in $Q_{j}$. We can proceed as in the preceding lemma to get $\lim _{j \rightarrow \infty} Q_{j}=0, \mathbb{P}$ almost surely. Now we use the upper bound to obtain for $j$ large

$$
R_{j} \leq C \sqrt{l(j)}\left(\sum_{k=j-2 l(j)}^{j-l(j)} \operatorname{card}\left\{q_{j}\left(\underline{s}_{k}\right) 2^{3(k-j) d / 2}\right\}\right)
$$

(recall $|s|=j-l(j)$ and $\left|\mu_{u, l, r}\right| \leq 2^{3(|r|-j) d / 2}$ ) and then $R_{j} \leq C \sqrt{l(j)} / j$, hence the lemma.

It remains to estimate

$$
B_{j}=\left(\sum_{l, r} \sum_{u \in q_{j}\left(\underline{s}_{j-2 L j}\right)} \mu_{u, l, r}\right) S\left(\underline{s}_{j-2 L j}\right) .
$$

As the summation in $r$ is reduced to $r \prec \underline{s}_{j-2 L j}$, we get $B_{j}=S\left(\underline{s}_{j-2 L j}\right)$ $j\left(1+\varepsilon_{j}\right) / j$ where $\varepsilon_{j} \rightarrow 0$.

The previous Lemmas and estimations give us

$$
\eta_{j}^{\varepsilon}(s)=\frac{S\left(\underline{s}_{j-2 L j}\right)}{j}\left(1+T_{j}\right)
$$

with $\lim _{j \rightarrow \infty} T_{j}=0, \mathbb{P}$ almost surely. and the lower bound is now a direct consequence of the following lemma

\section{Lemma 3.7.}

$$
\limsup _{j \rightarrow \infty} \max _{|s|=j-L j}\left|\tilde{\eta}_{j}(s)\right|=\sqrt{2 d \log 2} .
$$

Proof. The random variables $\sqrt{j} \tilde{\eta}_{j}(s)$ are independant Gaussian centered and of variance 1; so that the lemma is a classical asymptotic result, see [32] for instance. 


\subsection{A corollary.}

We can identify the $2^{d}$-adic tree $\delta$ and $D$ the set of dyadic points in $(0,1)^{d}$. Let $c$ be a continuous function on $(0,1)^{d}$, and $\left(\xi_{t}\right)_{t \in D y}$ an i.i.d. family of centered Gaussian random variables such that $\operatorname{var}\left(\xi_{t}\right)=c(t)$. We define the process $Z_{t}$ by

$$
Z_{t}=\sum_{s \in C_{t}^{*}} \xi_{t}
$$

Let $\bar{c}=\max _{t \in(0,1)^{d}} c(t)$.

\section{Proposition 3.2.}

$$
\limsup _{j \rightarrow \infty} \frac{1}{j} \max _{|t|=j}\left|Z_{t}\right|=\bar{c} \sqrt{2 d \log 2},
$$

$\mathbb{P}$ almost surely.

Proof. The upper and lower bounds of the previous demonstration, $c \equiv 1$, can be adapted to the present case. We need only to change the constant of Lemma (3.7) which becomes $\bar{c} \sqrt{2 d \log 2}$.

\section{Regularity of Elliptic Gaussian Processes.}

In this part we prove Theorems 1.3 and 1.7. Recall that here $m>d$ (then $X_{A}$ is an ordinary Gaussian process), $l \in \mathbb{N}$ and $\alpha \in(0,1]$ are the numbers defined by $(m-d) / 2=l+\alpha$. Recall also that we can suppose that HAS $(m, \gamma)$ hold.

We begin with the proof of Theorem 1.3. For the results of this section the process $X_{A}$ is restricted to a bounded domain $D$. Without loss of generality, we suppose that $D=(0,1)^{d}$. We prove first the law of the uniform modulus with $l=0, \alpha=1$, then we study the case $l=0$, we prove the law of iterated logarithm (local modulus), when $\alpha=1$ and also when $\alpha<1$. Finally we explain how to get the results without restrictions on $l$.

As explained in Section 1, we will use the decomposition of $X_{A}$ on a wavelet orthonormal basis of $H_{A}$. We introduce therefore a few more notations. If $\left\{\Phi_{\lambda}, \lambda \in \Lambda\right\}$ is the wavelet basis of the Hilbert space $H_{A}$, given by Theorem 1.1, for each $f \in H_{A}$,

$$
f=\sum_{\lambda \in \Lambda} f_{\lambda} \Phi_{\lambda}, \quad \text { with } f_{\lambda}:=\mathcal{A}\left(f, \Phi_{\lambda}\right) .
$$


If $g$ denotes a strictly increasing function of $\mathbb{N}$ in $\mathbb{R}_{+}$(which will be later chosen), we define the functions $\tilde{f}, f_{n}$, with $\lambda=(j, k, l)$, by

$$
\begin{gathered}
\tilde{f}(x)=\sum_{j \geq 0} \sum_{|x-\lambda| \leq 2^{-j} g(j) \sqrt{j}} \Phi_{\lambda}(x) f_{\lambda}, \\
f_{n}(x)=\sum_{0 \leq j \leq n} \Phi_{\lambda}(x) f_{\lambda},
\end{gathered}
$$

and in addition,

$$
\tilde{R} f=f-\tilde{f}, \quad R_{n} f=f-f_{n} .
$$

We need another operation which will perform averages. Recall that $c_{\lambda}$ is the dyadic cell with center $\lambda$ and side length $2^{-j}$. For $f \in L_{\mathrm{loc}}^{1}\left(R^{d}\right)$, let

$$
\bar{f}(\lambda)=2^{j d} \int_{c_{\lambda}} f(x) d x
$$

\subsection{Law of the uniform modulus when $l=0, \alpha=1$.}

The main idea is to make reductions in order to be able to use Lemma 3.1 and its corollary. This is done with the help of the projectors defined above. Let $\tilde{X}_{n}$ be the process defined by (71), (72) and define

$$
\Delta_{n}=\left\{(x, y) \in D \times D: 2^{n-1}<|x-y| \leq 2^{-n}\right\} .
$$

Let us explain the reductions we plan to do.

First reduction. We will prove

$$
\begin{aligned}
\limsup _{n \rightarrow \infty} \max _{(x, y) \in \Delta_{n}} & \frac{\left|\tilde{X}_{n}(x)-\tilde{X}_{n}(y)\right|}{|x-y| \sqrt{\log |x-y|^{-1}}} \\
& =\limsup _{n \rightarrow \infty} \max _{(x, y) \in \Delta_{n}} \frac{|X(x)-X(y)|}{|x-y| \sqrt{\log |x-y|^{-1}}},
\end{aligned}
$$

$\mathbb{P}$ almost surely. That is,

$$
\limsup _{n \rightarrow \infty} \max _{(x, y) \in \Delta_{n}} \frac{\left|R_{n} X(x)-R_{n} X(y)\right|}{|x-y| \sqrt{\log |x-y|^{-1}}}=0,
$$


$\mathbb{P}$ almost surely, and

$$
\limsup _{n \rightarrow \infty} \max _{(x, y) \in \Delta_{n}} \frac{\left|\tilde{R} X_{n}(x)-\tilde{R} X_{n}(y)\right|}{|x-y| \sqrt{\log |x-y|^{-1}}}=0,
$$

$\mathbb{P}$ almost surely.

Second reduction. In order to describe this second reduction we must first introduce some additional notations. For $n \in \mathbb{N}$ let us define $\bar{n} \in \mathbb{N}$ and the set $\bar{\Lambda}_{n} \subset \Lambda$ by

$$
\bar{n}=\left[\log \frac{2^{n}}{g(n) \sqrt{n}}\right], \quad \bar{\Lambda}_{n}=\left\{\lambda \in \Lambda: j_{\lambda}=\bar{n}\right\} .
$$

Now if $\mu \in(0,1)$, the integers $n_{\mu}$ and $m_{\mu}$ and the set $\Lambda_{n}^{\mu}$ are defined by

$$
n_{\mu}=[(1-\mu) n], \quad m_{\mu}=[\mu n], \quad \Lambda_{n}^{\mu}=\left\{\lambda \in \Lambda: j_{\lambda}=m_{\mu}\right\} .
$$

Denote by $\bar{Q}_{n}$ (respectively $Q_{n}^{\mu}$ ) the set of dyadic cells $\left\{\bar{c}_{\lambda}, j_{\lambda}=\bar{n}\right\}$ (respectively $\left\{c_{n}^{\mu}, j_{\lambda}=m_{\mu}\right\}$ ). In a $c_{n}^{\mu}$-cell there are $2^{\bar{n}-m_{\mu}} \bar{c}_{n}$-cells.

REMARK 5.1. Let $n^{0}$ be the integer defined by

$$
\frac{\log \left(g\left(n^{0}\right)^{2} n^{0}\right)}{n^{0}}<2 \mu<\frac{\log \left(g\left(n^{0}+1\right)\right)^{2}\left(n^{0}+1\right)}{n^{0}+1},
$$

then $\bar{n}>m_{\mu}$, for all $n \geq n^{0}$.

When $\left(x, x^{\prime}\right) \in \Delta_{n},\left(y, y^{\prime}\right) \in \Delta_{n}$ and $|x-y|>2^{-m_{\mu}}$, the random variables $\left(\tilde{X}_{n}(x)-\tilde{X}_{n}\left(x^{\prime}\right)\right)$ and $\left(\tilde{X}_{n}(y)-\tilde{X}_{n}\left(y^{\prime}\right)\right)$ are conditionally independent knowing $\sigma\left\{\xi_{\lambda}, j_{\lambda} \leq n_{0}\right\}$. Now for every $\lambda \in \Lambda$, the neighbourhood $\vee(\lambda)$ of $\lambda$ is defined by

$$
\vee(\lambda)=\left\{\lambda^{\prime} \in \Lambda: j_{\lambda}=j_{\lambda^{\prime}} \text { and } \partial c_{\lambda^{\prime}} \cap \partial c_{\lambda} \neq \varnothing\right\} .
$$

Then if $\tilde{\tilde{X}}_{n}(\lambda)$ is defined as in (74), let $d_{n}(\lambda)$ be defined by

$$
d_{n}(\lambda)=\max _{\lambda^{\prime} \in \vee(\lambda)} \frac{\left|\tilde{\tilde{X}}_{n}(\lambda)-\overline{\tilde{X}}_{n}\left(\lambda^{\prime}\right)\right|}{\left|\lambda-\lambda^{\prime}\right|} .
$$

Thanks to Remark 5.1, we are now in the situation of applying Proposition 3.2 and the second reduction consists in proving that

$$
\begin{aligned}
\limsup _{n \rightarrow \infty} \frac{1}{n} \max _{\lambda \in \bar{\Lambda}_{n}} d_{n}(\lambda) \\
\quad=\limsup _{n \rightarrow \infty} \max _{(x, y) \in \Delta_{n}} \frac{\left|\tilde{X}_{n}(x)-\tilde{X}_{n}(y)\right|}{|x-y| \sqrt{\log |x-y|^{-1}}},
\end{aligned}
$$


$\mathbb{P}$ almost surely, and

$$
\limsup _{n \rightarrow \infty} \frac{1}{n} \max _{\lambda \in \bar{\Lambda}_{n}} d_{n}(\lambda)=\sqrt{2 d} C_{D}
$$

$\mathbb{P}$ almost surely, with

$$
C_{D}^{2}=\limsup _{\substack{x, t \in D \\|x-y| \rightarrow 0}} \frac{\mathbb{E}\left[(X(x)-X(y))^{2}\right]}{|x-y|^{2} \log |x-y|^{-1}} .
$$

Step 1. Proof of (84).

As we have seen, if $j_{\lambda}=m_{\mu}$ there is $2^{\bar{n}-m_{\mu}}$ cells of $\bar{Q}_{n}$ in each $q_{\lambda}^{\mu}$ cell. Let $K_{n}^{\mu}$ be the set $\left\{1, \ldots, 2^{\bar{n}-m_{\mu}}\right\}$ and if $\lambda \in \Lambda_{n}^{\mu}$ let $i(\lambda)$ be the position in $c_{n}^{\mu}$ of the $i^{r d}$ cell $c_{\lambda}^{\mu}$ of $\bar{Q}_{n} \cap c_{n}^{\mu}$ (with the abuse $\bar{Q}_{n} \cap c_{n}^{\mu}=\left\{c \in \bar{Q}_{n}\right.$ : exists $\tilde{c} \in c_{n}^{\mu}$ and $\left.\left.c \subset \tilde{c}\right\}\right)$. Let

$$
\sigma^{2}\left(\lambda, \lambda^{\prime}\right)=\mathbb{E}\left[\frac{\left(\tilde{\tilde{X}}_{n}(\lambda)-\overline{\tilde{X}}_{n}\left(\lambda^{\prime}\right)\right)^{2}}{\left|\lambda-\lambda^{\prime}\right|^{2} \log \left|\lambda-\lambda^{\prime}\right|}\right]
$$

and

$$
\sigma^{2}(\lambda)=\max _{\lambda^{\prime} \in \vee(\lambda)} \sigma^{2}\left(\lambda, \lambda^{\prime}\right)
$$

On the other hand, let us define functions $\left\{\phi_{k}\left(\lambda, \lambda^{\prime}\right), k=1, \ldots, n\right\}$ and random variables $\left\{\eta_{k}(\lambda), k=1, \ldots, n\right\}$ such that

$$
\begin{aligned}
\frac{\left(\overline{\tilde{X}}_{n}(\lambda)-\overline{\tilde{X}}_{n}\left(\lambda^{\prime}\right)\right)}{\left|\lambda-\lambda^{\prime}\right|} & =\sum_{k \leq n} \sum_{|x-r| \leq 2^{-k d} g(k) \sqrt{k}}\left(\frac{\bar{\Phi}_{r}(\lambda)-\bar{\Phi}_{r}\left(\lambda^{\prime}\right)}{\left|\lambda-\lambda^{\prime}\right|}\right) \xi_{r} \\
& :=\sum_{k \leq n} \phi_{k}\left(\lambda, \lambda^{\prime}\right) \eta_{k}(\lambda) .
\end{aligned}
$$

Let $n^{0}$ be the integer of Remark 5.1. It is clear that $\left\{\eta_{\lambda}: j_{\lambda}>n_{0}\right\}$ is an i.i.d. family of Gaussian normal random variables. Furthermore,

$$
\sum_{k=1}^{n} \phi_{k}^{2}\left(\lambda, \lambda^{\prime}\right)=\sigma^{2}\left(\lambda, \lambda^{\prime}\right) .
$$

Hence, if $\mu$ is fixed and $n$ is large enough we will be in the situation of Proposition 3.2. Therefore, for every sequence $\left\{i_{n}\right\}_{n \geq 0}$ such that $i_{n} \in K_{n}^{\mu}$

$$
\limsup _{n \rightarrow \infty} \frac{1}{n} \max _{|\nu|=n_{\mu}} d_{n}\left(i_{n}(\nu)\right)=\sqrt{(1-\mu) 2 \log d} C_{D},
$$


$\mathbb{P}$ almost surely. Let $\mu \rightarrow 0$, we obtain

$$
\limsup _{n \rightarrow \infty} \frac{1}{n} \max _{j_{\lambda}=n} d_{n}(\lambda) \geq \sqrt{2 d} C_{D}
$$

$\mathbb{P}$ almost surely. Since the upper bound is easily deduced from the one of Proposition 3.2, we have proved (84).

Step 2. Proof of (83).

Here we must go from averages to pointwise values. Let

$$
S\left(\lambda, \lambda^{\prime} ; x, y\right)=\frac{1}{n} d_{n}\left(\lambda, \lambda^{\prime}\right)-\frac{\tilde{X}_{n}(x)-\tilde{X}_{n}(y)}{|x-y| \sqrt{\log |x-y|^{-1}}},
$$

for $x \in c_{\lambda},(x, y) \in \Delta_{n}$. In order to prove (83) it is enough to show that,

$$
\limsup _{n \rightarrow \infty} \max _{\substack{\lambda \in \Lambda_{n} \\ \lambda^{\prime} \in \vee(\lambda) \\ x \in c_{\lambda} \\(x, y) \in \Delta_{n}}}\left|S\left(\lambda, \lambda^{\prime} ; x, y\right)\right|=0
$$

$\mathbb{P}$ almost surely, but

$$
\begin{array}{r}
\left|S\left(\lambda, \lambda^{\prime} ; x, y\right)\right| \\
=\frac{1}{n} \mid \sum_{k \leq n} \sum_{\substack{j_{r}=k \\
2^{k}|r-\lambda| \leq g(k) \sqrt{k}}}\left(2^{n d} \int_{c_{\lambda}}\left(\frac{\Phi_{r}\left(x^{\prime}\right)-\Phi_{r}(x)}{2^{-n}}\right) d x^{\prime}\right. \\
\\
\left.-2^{n d} \int_{c_{\lambda}}\left(\frac{\Phi_{r}\left(y^{\prime}\right)-\Phi_{r}(y)}{2^{-n}}\right) d y^{\prime}\right) \xi_{r} \mid .
\end{array}
$$

On the other hand,

$$
\begin{aligned}
\int_{c_{\lambda}}\left(\Phi_{r}\left(x^{\prime}\right)-\Phi_{r}(x)\right) d x^{\prime}- & \int_{c_{\lambda}}\left(\Phi_{r}\left(y^{\prime}\right)-\Phi_{r}(y)\right) d y^{\prime} \\
& =C\left(2^{n d} \int_{c_{\lambda}} D^{2} \Phi_{r}\left(\lambda, \lambda^{\prime}\right)\left(d x, d x^{\prime}\right)+\varepsilon\right),
\end{aligned}
$$

for $C>0$. Denote by $A_{r}$ this quantity; using Theorem 1.1,

$$
\left|A_{r}\right| \leq C 2^{-n} 2^{(d / 2-m / 2+2) r} 2^{-2 n}=C 2^{(d / 2-m / 2-1) n} .
$$


Thanks to a result proved in [3] we have

$$
\sum_{2^{k}|\lambda-r| \leq g(k) \sqrt{k}}\left|A_{r}\right| \leq 4 n
$$

if $n$ is large enough. So

$$
\left|S\left(\lambda, \lambda^{\prime} ; x, y\right)\right| \leq 2 C \frac{2^{-n}}{n} \sum_{k \leq n} \sqrt{k} 2^{k} 2^{(d / 2-m / 2-1) n}
$$

if $n$ is large enough. Then (83) follows.

Step 3. Proof of (76).

It is sufficient to prove (77) and (78). Let us begin by (78). Taking into account that $\left|\xi_{r}\right| \leq \sqrt{2} \sqrt{r}$ if $|r|$ is large enough, and that

$$
\sum_{|l| \geq g(k) \sqrt{k}} \frac{1}{(1+|l|)^{d+1}} \simeq \frac{1}{g(k) \sqrt{k}},
$$

(78) becomes

$$
\frac{\left|\tilde{R} X_{n}(s)-\tilde{R} X_{n}(y)\right|}{|x-y| \sqrt{\log |x-y|^{-1}}} \leq \frac{1}{n} \sum_{1}^{n} \frac{1}{g(k)},
$$

but with a correct choice of function $g$ we can deduce (78).

To prove (77) we use the same method as above. For $\beta>0$ using again Theorem 1.1, (77) becomes

$$
\begin{aligned}
\frac{\left|R_{n} X(x)-R_{n} X(y)\right|}{|x-y| \sqrt{\log |x-y|^{-1}}} & \leq \frac{1}{n} \sum_{k=n}^{n+\beta \log n} \sqrt{k}+2 \frac{2^{n}}{n} \sqrt{k} 2^{-k} \\
& \leq \frac{2}{n \beta}+\frac{3}{2} \beta \frac{\log n}{\sqrt{n}}
\end{aligned}
$$

if $n$ is large enough; in the first part of the proof of the upper bound we have used (2.2) with $|\alpha|=1$ and with $|\alpha|=0$ in the second one, where furthermore the inequality $|x-y|<|x|+|y|$ has been used, hence the factor 2 . 
Conclusion. The proof of the law of the uniform modulus (30) results then from (84), (83) and (76).

\subsection{Law of the uniform modulus when $l=0,0<\alpha<1$.}

In the present case, the reductions we perform will lead to a Brownian motion-like situation ( $c f$. Introduction) or more precisely [3]-like situation. We set $\ell_{\alpha}(r)=r^{\alpha}$.

First reduction. Let $\beta>0$, let us introduce the integers $n_{\beta}^{ \pm}=n \pm$ $[\beta \log n]$. We have to prove

$$
\limsup _{n \rightarrow \infty} \max _{(x, y) \in \Delta_{n}} \frac{\left|X_{n_{\beta}^{-}}(x)-X_{n_{\beta}^{-}}(y)\right|}{\ell_{\alpha}(|x-y|)}=0,
$$

$\mathbb{P}$ almost surely, and

$$
\limsup _{n \rightarrow \infty} \max _{(x, y) \in \Delta_{n}} \frac{\left|R_{n_{\beta}^{+}} X(x)-R_{n_{\beta}^{+}} X(y)\right|}{\ell_{\alpha}(|x-y|)}=0,
$$

$\mathbb{P}$ almost surely. That is to say, low and high scales have no contribution to the result. Let

$$
S_{n}(x, y)=\left(R_{n_{\beta}^{-}} X(x)-R_{n_{\beta}^{-}} X(y)\right)-\left(R_{n_{\beta}^{+}} X(x)-R_{n_{\beta}^{+}} X(y)\right),
$$

corresponding to the terms of scale between $n_{\beta}^{-}$and $n_{\beta}^{+}$;

$$
\limsup _{n \rightarrow \infty} \max _{(x, y) \in \Delta_{n}} \frac{\left|S_{n}(x, y)\right|}{l_{\alpha}(|x-y|)}=\limsup _{(x, y) \in \Delta_{n}} \frac{|X(x)-X(y)|}{l_{\alpha}(|x-y|)}
$$

$\mathbb{P}$ almost surely, is a consequence of (86) and (87).

Second reduction. The second reduction will lead to a situation where the wavelets will be thought of as compactly supported. We have to show that

$$
\limsup _{n \rightarrow} \max _{(x, y) \in \Delta_{n}} \frac{\left|S_{n}(x, y)\right|}{l_{\alpha}(|x-y|)}=\limsup _{(x, y) \in \Delta_{n}} \frac{\left|\tilde{S}_{n}(x, y)\right|}{l_{\alpha}(|x-y|)},
$$


$\mathbb{P}$ almost surely, where $\tilde{S}(x, y)$ is obtained by applying the operator (71) in each variable $x$ and $y$.

Third reduction. The third reduction consists in defining a sequence of partitions $\left(P_{n}\right)_{n \geq 0}$ of the domain $D$ such that if $q$ and $q^{\prime}$ are two elements of $P_{n}$ sufficiently far away then $\left\{\tilde{S}_{n}(x, y):(x, y) \in q \times q\right\}$ and $\left\{\tilde{S}_{n}(x, y):(x, y) \in q^{\prime} \times q^{\prime}\right\}$ become independent.

The integer $\bar{n}$ is, as before, equal to $\left[\log \left(2^{n} / g(n) \sqrt{n}\right)\right]$. When $s>0$ let $\bar{n}_{s}=\bar{n} / n^{s}$. Consider the subsets

$$
\bar{\Lambda}_{n}=\left\{\lambda \in \Lambda: j_{\lambda}=\bar{n}\right\}, \quad \bar{\Lambda}_{n}^{s}=\left\{\lambda \in \Lambda: j_{\lambda}=\bar{n}_{s}\right\} .
$$

Looking for the cells $\bar{q}_{\lambda}$ and $q_{\lambda}^{s}$ if $\lambda \in \bar{\Lambda}_{n}$ and $\lambda \in \bar{\Lambda}_{n}^{s}$ respectively, we define $\tilde{q}_{\lambda}^{s}$ as the cell with the same center as $q_{\lambda}^{s}$, with faces parallel to the axes and with side lengths $2^{-n}\left(\bar{n}-\bar{n}_{s}\right)$. In these conditions, for all $s>0$, by construction we have independence between $\sigma\left\{\tilde{S}_{n}(x, y)\right.$ : $\left.(x, y) \in \tilde{q}_{\lambda}^{s} \times \tilde{q}_{\lambda}^{s}\right\}$ and $\sigma\left\{\tilde{S}_{n}(x, y):(x, y) \in \tilde{q}_{\lambda}^{\prime}(x, y) \times \tilde{q}_{\lambda}^{\prime}(x, y)\right\}$ for $\lambda \neq \lambda^{\prime}$, $j_{\lambda}=j_{\lambda^{\prime}}=\bar{n}_{s}$.

Now let $\vee(\lambda)$ as before the set of neighbours of $\lambda$. We have to show that

$$
\begin{aligned}
\limsup _{n \rightarrow \infty} \max _{\substack{j_{\lambda}=\bar{n}-s \log \bar{n} \\
\lambda^{\prime} \in \vee(\lambda)}} \frac{\left|\bar{S}_{n}\left(\lambda, \lambda^{\prime}\right)\right|}{2^{-\alpha n \sqrt{n d \log 2}}} \\
\quad=\limsup _{n \rightarrow \infty} \max _{(x, y) \in \Delta_{n}} \frac{\left|\tilde{S}_{n}(x, y)\right|}{l_{\alpha}(x, y)},
\end{aligned}
$$

$\mathbb{P}$ almost surely, and this is a consequence of

$$
\limsup _{n \rightarrow \infty} \max _{\substack{j_{\lambda}=\bar{n}-s \log \bar{n} \\(x, y) \in \Delta_{n}}}\left|\frac{\bar{S}_{n}\left(\lambda, \lambda^{\prime}\right)}{2^{-\alpha n} \sqrt{n d \log 2}}-\frac{\bar{S}_{n}(x, y)}{l_{\alpha}(|x-y|)}\right|=0,
$$

$\mathbb{P}$ almost surely. The proof of (90) is in every way analogous to the one of (83). Now the method of [3] can be directly used for showing

$$
\limsup _{n \rightarrow \infty} \max _{j_{\lambda}=\bar{n}-s \log \bar{n}} \frac{\left|\bar{S}_{n}\left(\lambda, \lambda^{\prime}\right)\right|}{2^{-d n} \sqrt{n}}=\sqrt{d \log 2} C_{D}
$$

$\mathbb{P}$ almost surely, and also (86), (87). For the last results we use well known bounds for independant gaussian random variables and the inequalities (18), (19). 


\subsection{Law of uniform "Zygmund-class"-modulus when $l=0$, $\alpha=1$.}

We can proceed as in the last paragraph, using (18), (19) to restrict the sum only to scales $j_{\lambda} \sim \log (|x-y|)$. Following the method of [3] the above reductions give the result.

\subsection{Law of the iterated logarithm when $l=0, \alpha=1$.}

Here we set $l_{1}^{(2)}(r)=|r| \sqrt{\log r^{-1} \log \log \log r^{-1}}$. To prove that for $y \in D$ we have

$$
\limsup _{x \rightarrow y} \frac{|X(x)-X(y)|}{l_{1}^{(2)}(|x-y|)}=\sqrt{2} C(y),
$$

$\mathbb{P}$ almost surely. We consider reductions of the problem absolutely similar to the preceding ones. We will also use the well known result of Levy-Kinchin,

$$
\limsup _{n \rightarrow \infty} \frac{1}{\sqrt{n \log \log n}}\left|\sum_{k=1}^{n} \xi_{k}\right|=\sqrt{2}
$$

$\mathbb{P}$ almost surely, where $\left(\xi_{k}, k \in \mathbb{N}\right)$ is an i.i.d. sequence of Gaussian normal random variables.

Hence, using the modulus $l_{1}^{2}(\rho)$ in place of $l_{1}(r)=|r| \log \left(r^{-1}\right)$ it is possible to prove an inequality analogous to (77) and (78), therefore an equality similar to (76). In these conditions (83) will become

$$
\begin{aligned}
\limsup _{n \rightarrow \infty} \frac{1}{\sqrt{n \log \log n}} & \left(\max _{\lambda \in \bar{\Lambda}_{k}(y)} d_{n}(\lambda)\right) \\
& =\limsup _{n \rightarrow \infty} \max _{(x, y) \in \Delta_{n}} \frac{\left|\tilde{X}_{n}(x)-\tilde{X}_{n}(y)\right|}{l_{1}^{(2)}(|x-y|)}
\end{aligned}
$$

where $\bar{\Lambda}_{k}(y)=\left\{\lambda \in \bar{\Lambda}_{k}\right.$ and $\left.2^{n} d(\lambda, y) \leq g(n) \sqrt{n}\right\}$.

Then using (91) the first member of (92) converges to $\sqrt{2} C(y)$ almost surely. 
4.5. Law of the iterated logarithm when $l=0,0<\alpha<1$.

To prove the law of the iterated logarithm we have only to show that

$$
\limsup _{x \rightarrow y} \frac{|X(x)-X(y)|}{l_{\alpha}^{(2)}(|x-y|)}=\limsup _{n \rightarrow \infty} \frac{1}{\sqrt{\log n}} \max _{(x, y) \in \Delta_{n}} K,
$$

$\mathbb{P}$ almost surely, with

$$
K=2^{\alpha n} \sum_{\substack{n-\beta \log \log n<k<n+\beta \log \log n \\ 2^{k}|y-r|<g(k) \sqrt{k}}}\left(\Phi_{r}(x)-\Phi_{r}(y)\right) \xi_{r} \mid \sqrt{2 \log 2} C_{s}(g) .
$$

The same reductions as above show that we can use the proof of the same result given in [BJR] for the one dimensional case. Hence (93).

\subsection{The laws of moduli when $l \neq 0$.}

In order to end the proof of Theorem 1.3 we still have to consider the case $l \neq 0$.

Let $s$ be a multi-index of length $l$. Let us set $Y(x)=\partial^{s} X(x)$. Thanks to Proposition 2.1,

$$
Y(x)=\sum_{\lambda \in \Lambda} \partial^{s} \Phi_{\lambda}(x) \xi_{\lambda}: \simeq \sum_{\lambda \in \Lambda} \theta_{\lambda} \xi_{\lambda} .
$$

Let $\tilde{A}$ be the elliptic operator defining the topology of the auto-reproducing Hilbert space $H_{y}$ of $Y$. As $\theta_{\lambda}$ is an orthonormal basis of $H_{y}$, it follows that $\theta_{\lambda}=\tilde{A}^{-1 / 2} \psi_{\lambda}$; as $\partial^{s} \varphi_{\lambda}=\partial^{s} A^{-1 / 2} \psi_{\lambda}$, we get $\tilde{A}^{-1 / 2}=$ $\partial^{s} A^{-1 / 2}$. So, the symbol $\sigma_{y}$ of $\tilde{A}$ is of degree $m-2 l$; it satisfies Hypothesis HA $(m-2 l, \gamma)$. Therefore, performing the same calculus as above we obtain the theorem in the general case.

\subsection{Approximation of elliptic gaussian generalized processes.}

Let us now prove Theorem 1.6 which concerns generalized Gaussian processes. From the estimations of Theorem 1.1 we know there exists $r_{d}>0$ such that

$$
\text { i) } \lim _{h \rightarrow 0} \sum_{0 \leq j_{\lambda} \leq \log _{2}\left(h^{-1}\right)} \frac{\left(\Phi_{\lambda}(x+h)-\Phi_{\lambda}(x)\right)^{2}}{|h|^{2} \log \left(|h|^{-1}\right)}=r_{d}^{2},
$$


when $d=1$ or $d=2$, and

$$
\text { ii) } \lim _{h \rightarrow 0} \sum_{0 \leq j_{\lambda} \leq \log _{2}\left(h^{-1}\right)} \frac{\left(\Phi_{\lambda}(x+h)-\Phi_{\lambda}(x)\right)^{2}}{|h|^{2}}=r_{d}^{2},
$$

if $d \geq 3$. Using these limits we can transpose the proofs we gave for the law of the uniform modulus to get the results of Theorem 1.6. Note that when $d=1, d=2$ then $\alpha=1$ (critical case) so that $C_{d}=r_{d} \sqrt{2 \log 2}$. When $d \geq 3, \alpha=1 / 2$ and $C_{d}=r_{c} \sqrt{2 d \log 2}$.

\subsection{Moduli of continuity for the multifractional Brownian motion.}

We constructed a collection of wavelets $\omega_{\lambda}$ which, because of the decomposition (44), plays for the multifractional Brownian motion exactly the same role as the $\Phi_{\lambda}$ for Elliptic Processes. The proofs of regularity results for the multifractional Brownian motion are similar to [3] and we will just sketch them. We first prove "vaguelettes-type" localization estimates for the $\omega_{\lambda}$ defined in (43).

Proposition 4.1. We assume the function a belongs to $C^{r}\left(\mathbb{R}^{d},(0,1)\right)$, $\sup a(x) \leq r, r>0,(j, l) \neq(0,0)$ and $K \in \mathbb{N}$. Then there exists $a$ constant $C$ (which depends on $K$ ) such that

$$
\left|\omega_{\lambda}(x)\right| \leq C 2^{-j a(x)}\left(\frac{1}{\left(1+\left|2^{j} x-k\right|\right)^{K}}+\frac{1}{(1+|k|)^{K}}\right)
$$

and

$$
\left|\omega_{\lambda}(x)-\omega_{\lambda}(y)\right|
$$

$$
\leq C 2^{-j a(x)}\left(\frac{2^{j}|x-y|+j|a(x)-a(y)|}{\left(1+\left|2^{j} x-k\right|\right)^{K}}+\frac{j|a(x)-a(y)|}{(1+|k|)^{K}}\right) .
$$

Proof. We want to bound

$$
H=\int \frac{e^{i x \xi}}{|\xi|^{a(x)+d / 2}} \hat{\psi}_{\lambda}(\xi) d \xi=2^{-j d / 2} \int \frac{e^{i(x-\lambda) \xi}}{|\xi|^{a(x)+d / 2}} \hat{\psi}^{(l)}\left(\frac{\xi}{2^{j}}\right) d \xi .
$$

Let us recall that the support of $\hat{\psi}$ is included in $\{\xi: 2 \pi / 3 \leq|\xi| \leq$ $8 \pi / 3\}$. Setting $\nu=\xi / 2^{j}$ in the integral we get easily

$$
|H| \leq c 2^{-j a(\lambda)} \text {. }
$$


If this change of variable is made after $K$ integrations by part in a direction where

$$
|x-\lambda| \leq d\left|x_{p}-\lambda_{p}\right|
$$

we get

$$
H \leq c 2^{-j(K+a(x))}|x-\lambda|^{-K} .
$$

From these two inequalities we deduce (96).

For the second result we write $\omega_{\lambda}(x)-\omega_{\lambda}(y)=R+S$, where

$$
R=2^{-j d / 2} \int \frac{e^{i(x-\lambda) \xi}\left(e^{i(y-x) \xi}-1\right)}{|\xi|^{a(x)+d / 2}} \hat{\psi}^{(l)}\left(\frac{\xi}{2^{j}}\right) d \xi
$$

and

$$
S=2^{-j d / 2} \int \frac{\left(e^{i(y) \xi}-1\right) e^{-i \xi \lambda}}{|\xi|^{a(x)+d / 2}}\left(\frac{1}{|\xi|^{a(y)-a(x)}}-1\right) \hat{\psi}^{(l)}\left(\frac{\xi}{2^{j}}\right) d \xi
$$

To give a bound for $R$ we use $\left|e^{i h \xi}-1\right| \leq|h||\xi|$ and proceed as in the proof of (96), so that

$$
|R| \leq c 2^{-j a(x)} \frac{2^{j}|x-y|}{\left(1+\left|2^{j} x-k\right|\right)^{K}}
$$

Now we can split $S$ as $S=S_{1}+S_{2}$,

$$
\begin{gathered}
S_{1}=2^{-j(a(x)+d)} \int \frac{\left(e^{i(y) \xi}-1\right) e^{-i \xi \lambda}}{\left|\xi / 2^{j}\right|^{a(x)+d / 2}}\left(\frac{1}{\left|\xi / 2^{j}\right|^{a(y)-a(x)}-1}\right) \hat{\psi}^{(l)}\left(\frac{\xi}{2^{j}}\right) d \xi \\
S_{2}=2^{-j d}\left(2^{j(a(x)-a(y))}-1\right) \int \frac{\left(e^{i(y) \xi}-1\right) e^{-i \xi \lambda}}{\left|\xi / 2^{j}\right|^{a(y)+d / 2}} \hat{\psi}^{(l)}\left(\frac{\xi}{2^{j}}\right) d \xi
\end{gathered}
$$

With the same integrations by part, change of variable and using the inequality

$$
r^{a(y)-a(x)}-1=O\left(|a(y)-a(x)| \log r \max \left\{r^{a(x)}, r^{a(y)}\right\}\right),
$$

we obtain

$$
\left|S_{i}\right| \leq c 2^{-j a(x)} \frac{j|a(x)-a(y)|}{(1+|k|)^{K}},
$$

and then the last estimate of the Proposition holds. 
Let us now prove the law of the uniform modulus. We use the decomposition (44) in order to estimate $\left(B_{\alpha}(x+h)-B_{\alpha}(x)\right)$. Setting $n=\left[\log _{2}|h|^{-1}\right]$ (so that $2^{-n-1}<|h| \leq 2^{-n}$ ), we separate the sum into four terms $T_{i}$ which correspond to the cases

1) $j<n-\beta \log n$,

2) $j>n+\beta \log n$,

3) $n-\beta \log n \leq j \leq n+\beta \log n, j\left(a_{E}-a(\lambda)\right) \leq \delta \log j$,

4) $n-\beta \log n \leq j \leq n+\beta \log n, j\left(a_{E}-a(\lambda)\right) \geq \delta \log j$.

Using well known properties of an independant sequence of standard gaussian random variables as in [3], when $\beta\left(1-a_{E}\right)>1 / 2$, we get from (97)

$$
\lim _{h \rightarrow 0}|h|^{-a_{E}} T_{1}=0
$$

and from (96)

$$
\lim _{h \rightarrow 0}|h|^{-a_{E}} T_{2}=0 .
$$

In the same way we deduce also from (97) that

$$
\lim _{h \rightarrow 0}|h|^{-a_{E}} T_{3}=0
$$

The relevant contribution of the sum is given by $T_{4}$. Now, using the continuity of the function $C_{E}$ and proceeding as in [3], we get

$$
\limsup _{\substack{x, y \in E \\|x-y| \rightarrow 0}} \frac{\left|B_{a}(x)-B_{a}(y)\right|}{|x-y|^{a_{E}} \sqrt{\log 1 /|x-y|}}=C_{E} \sqrt{d},
$$

$\mathbb{P}$ almost surely. The proof of the law of the iterated logarithm follows exactly the corresponding proof for E.G.R.P. in the non-critical case.

The asymptotic self similarity of the Multifractional B.M. $B_{a}$ is a straightforward application of the following Proposition. We define

$$
\theta_{\lambda}=\int \frac{e^{i x \xi}-1}{|\xi|^{a(\lambda)+d / 2}} \hat{\psi}_{\lambda}(\xi) d \xi
$$


Proposition 4.2. If the function a belongs to $C^{r}\left(\mathbb{R}^{d},(0,1)\right),(r>0)$, we have the following asymptotic behavior

$$
\begin{aligned}
\left|\omega_{\lambda}(x)-\theta_{\lambda}(x)\right| \leq & c j|a(x)-a(\lambda)| \\
& \cdot 2^{-j \min \{a(x), a(\lambda)\}}\left(\frac{1}{\left(1+\left|2^{j} x-k\right|\right)^{K}}+\frac{1}{(1+|k|)^{K}}\right) .
\end{aligned}
$$

The proof is along the lines of Proposition 4.1.

\section{Scaling properties for Elliptic Gaussian processes.}

In this part Theorems 1.4, 1.5 and Proposition 1.3 are proved. Recall that we want to study the local scaling properties for Elliptic Gaussian processes. They will be connected them with scaling properties of the associated symbols or wavelets. Consider a point $x_{0}$ in $\mathbb{R}^{d}$ which remains fixed for the whole paragraph. The whole-scale Littlewood-Paley basis (of $L^{2}$ ) is denoted by $\left\{\psi_{\mu}\right\}_{\mu \in \underline{\Lambda}}$, where $\underline{\Lambda}=\mathbb{Z} \times \mathbb{Z}^{d} \times L$.

Let $s$ be a symbol on $\mathbb{R}^{d}$. We define when it makes sense the function $g_{\lambda}^{s}$ by its Fourier transform

$$
\hat{g_{\lambda}^{s}}(\xi)=\frac{\hat{\psi_{\lambda}}(\xi)}{\sqrt{s(x, \xi)}}
$$

\subsection{Scaling properties for elliptic symbols.}

We suppose here that the symbol $\sigma$ fullfills hypotheses HA $(m, \gamma)$. We consider only the case $m=d+2 \alpha, 0<\alpha \leq 1$.

For $\rho>0$ we set

$$
\sigma^{x_{0}}(x, \xi)=\sigma\left(x_{0}+x, \xi\right), \quad \sigma_{\rho}^{x_{0}}(x, \xi)=\rho^{m} \sigma^{x_{0}}\left(\rho x, \frac{\xi}{\rho}\right) .
$$

Using the scaling properties of $\left\{\psi_{\lambda}\right\}_{\lambda}$, we have

$$
g_{\lambda}^{\sigma_{\rho}^{x_{0}}}(x)=\rho^{-\alpha} g_{\lambda(\rho)}^{\sigma^{x_{0}}}(\rho x),
$$

if $\rho=2^{-p}, \lambda(\rho)=2^{-j-p}(k+l / 2)=\rho \lambda$. The extension to $\rho$ positive real is obvious. 

we get

Consequently, when $\left(\xi_{\lambda}\right)_{\lambda \in \underline{\Lambda}}$ is an i.i.d. standard gaussian family,

$$
\begin{aligned}
\rho^{-\alpha} \sum_{j_{\lambda} \geq 0}\left(g_{\lambda}^{\sigma^{x_{0}}}\left(x 2^{-p}\right)\right. & \left.-g_{\lambda}^{\sigma^{x_{0}}}(0)\right) \xi_{\lambda} \\
& =\sum_{j_{\lambda} \geq 0}\left(g_{\lambda(1 / \rho)}^{\sigma_{\rho}^{x_{0}}}(x)-g_{\lambda(1 / \rho)}^{\sigma_{\rho}^{x_{0}}}(0)\right) \xi_{\lambda} .
\end{aligned}
$$

This gives the following equality in law

$$
\begin{aligned}
\rho^{-\alpha} \sum_{j_{\lambda} \geq 0}\left(g_{\lambda}^{\sigma^{x_{0}}}\left(x 2^{-p}\right)\right. & \left.-g_{\lambda}^{\sigma^{x_{0}}}(0)\right) \xi_{\lambda} \\
& \stackrel{(\mathrm{d})}{=} \sum_{j_{\mu} \geq-p}\left(g_{\mu}^{\sigma_{\rho}^{x_{0}}}(x)-g_{\mu}^{\sigma_{\rho}^{x_{0}}}(0)\right) \xi_{\mu} .
\end{aligned}
$$

Lemma 5.1. With the above notations, the convergence and the limit of $\sigma_{\rho}^{x_{0}}(x, \xi)$ when $\rho \rightarrow 0^{+}$is independant of $x$. In case of convergence, the limit function $\theta$ satisfies, for all $\xi$ and $r>0$,

$$
\theta(r \xi)=r^{m} \theta(\xi)
$$

and also, for all $\xi$,

$$
c|\xi|^{m} \leq|\theta(\xi)| \leq C|\xi|^{m},
$$

where $c, C$ are the ellipticity constants given by hypothesis $\mathrm{HA}(m, \gamma)$ for the symbol $\sigma$.

Proof. We know from HA $(m, \gamma)$ that

$$
\rho^{m}\left|\sigma\left(x_{0}+\rho x, \frac{\xi}{\rho}\right)-\sigma\left(x_{0}, \frac{\xi}{\rho}\right)\right| \leq K \rho^{m}\left(1+\frac{|\xi|}{\rho}\right)^{m+\varepsilon^{\prime}}|\rho x|^{\varepsilon},
$$

with $\varepsilon>\varepsilon^{\prime} \geq 0$. This is bounded by $K(\rho+|\xi|)^{m+\varepsilon^{\prime}}|x|^{\varepsilon} \rho^{\varepsilon-\varepsilon^{\prime}}=o(\rho)$. The first assertion of the Lemma is now clear. The homogeneity property of the limit function is classical. And the last inequalities are deduced from

$$
c|\xi|^{m} \leq \sigma(z, \xi) \leq C|\xi|^{m}, \quad \text { if }|\xi| \geq R
$$


which are part of our hypothesis.

\subsection{Local scaling for processes.}

We define the scaling operators $R_{\alpha, \rho}$ when $\alpha<1, L_{\rho}$ when $\alpha=1$ by

$$
\begin{gathered}
R_{\alpha, \rho}(f)=\frac{f\left(x_{0}+\rho \cdot\right)-f_{\lambda}\left(x_{0}\right)}{\rho^{\alpha}} \\
L_{\rho}(f)=\frac{1}{\sqrt{\log (1 / \rho)}} \frac{f\left(x_{0}+\rho \cdot\right)-f_{\lambda}\left(x_{0}\right)}{\rho} .
\end{gathered}
$$

We suppose here that the symbol $\sigma$ fullfills hypotheses HA $(m, \gamma), H 1$. We consider $A=o p(\sigma)$ and $X$ the gaussian process associated with. According to Proposition 1.2 we can write

$$
X_{x}=\sum_{\lambda \in \Lambda} \xi_{\lambda} \Phi_{\lambda}(x)
$$

with $\xi_{\lambda}$ i.i.d. standard gaussian. We complete the family with $\xi_{\lambda}$, $j_{\lambda}<0$ keeping the i.i.d. property valid.

We say that the symbol $\sigma$ satisfies hypothesis $H\left(x_{0}\right)$ when

$$
\lim _{\rho \rightarrow 0^{+}} \sigma_{\rho}^{x_{0}}(0, \xi)=\theta(\xi), \quad \text { for almost every } \xi \in \mathbb{R}^{d} .
$$

In this case we set

$$
Y_{x}=\sum_{\lambda \in \underline{\Lambda}} \xi_{\lambda} g_{\lambda}^{\theta}(x)
$$

We can now state convergence in law $((\mathrm{d})-\mathrm{lim})$ and equality in law $(\stackrel{(\mathrm{d})}{=})$ for the locally scaled processes.

Lemma 5.2. We suppose that the symbol $\sigma$ of the E.G.P. $X$ fullfills $\mathrm{HA}(m, \gamma), H\left(x_{0}\right)$ and $\operatorname{HAS}(m, \gamma)$. If $\alpha<1$,

$$
\text { (d)- } \lim _{\rho \rightarrow 0^{+}} R_{\alpha, \rho} X=Y \text {, }
$$

and for all $\rho>0$,

$$
R_{\alpha, \rho} Y \stackrel{(\mathrm{d})}{=} Y .
$$


If $\alpha=1$, there exists a gaussian vector $G$ on $\mathbb{R}^{d}$ such that

$$
\text { (d)- } \lim _{\rho \rightarrow 0^{+}} L_{\rho} X=(\mathrm{d})-\lim _{\rho \rightarrow 0^{+}} L_{\rho} Y=(G \mid x) \text {. }
$$

Proof. Case $\alpha<1$. Let us give first the idea of the proof. We approximate

$$
R_{\alpha, \rho} X=\rho^{-\alpha} \sum_{j_{\lambda} \geq 0}\left(\Phi_{\lambda}\left(x_{0}+\rho \cdot\right)-\Phi_{\lambda}\left(x_{0}\right)\right) \xi_{\lambda}
$$

by

$$
\rho^{-\alpha} \sum_{j_{\lambda} \geq 0}\left(g_{\lambda}^{\sigma^{x_{0}}}(\rho \cdot)-g_{\lambda}^{\sigma^{x_{0}}}(0)\right) \xi_{\lambda} .
$$

But as far as the laws are concerned we know from (100) that the renormalization of the above process is equivalent to a shift on the scales. We obtain

$$
R_{\alpha, \rho} X \stackrel{(\mathrm{d})}{\sim} \sum_{j_{\mu} \geq \log _{2} \rho}\left(g_{\mu}^{\sigma_{\rho}^{x_{0}}}(x)-g_{\mu}^{\sigma_{\rho}^{x_{0}}}(0)\right) \xi_{\mu}
$$

As $\lim _{\rho \rightarrow 0^{+}} \log _{2} \rho=-\infty$ and the symbol $\sigma_{\rho}^{x_{0}}$ converges to $\theta$ (by hypothesis) we get (103)

$$
\text { (d)- } \lim _{\rho \rightarrow 0^{+}} R_{\alpha, \rho} X_{x}=\sum_{\lambda \in \underline{\Lambda}} \xi_{\lambda} g_{\lambda}^{\theta}(x)
$$

Now let us give the technical justifications for the three steps just described. For the first step we use the approximation of wavelets given by the Theorem 1.3, so that

$$
\lim _{\rho \rightarrow 0^{+}} \rho^{-\alpha} \sum_{j_{\lambda} \geq 0}\left(g_{\lambda}^{\sigma^{x_{0}}}(\rho x)-g_{\lambda}^{\sigma^{x_{0}}}(0)\right) \xi_{\lambda}=0
$$

uniformly on every bounded set, $\mathbb{P}$ almost surely.

In the second step we apply directly (100) so that

$$
\sum_{j_{\lambda} \geq 0}\left(g_{\lambda}^{\sigma^{x_{0}}}\left(x 2^{-p}\right)-g_{\lambda}^{\sigma^{x_{0}}}(0)\right) \xi_{\lambda} \stackrel{(\mathrm{d})}{=} \sum_{j_{\mu} \geq-p}\left(g_{\mu}^{\sigma_{\rho}^{x_{0}}}(x)-g_{\mu}^{\sigma_{\rho}^{x_{0}}}(0)\right) \xi_{\mu} .
$$


For the last step we use the convergence of symbols given by hypothesis $H\left(x_{0}\right)$ and also Lemma 5.1. Then

$$
\text { (d)- } \lim _{\rho \rightarrow 0^{+}} \sum_{j_{\mu} \geq-\log _{2} \rho}\left(g_{\mu}^{\sigma_{\rho}^{x_{0}}}(x)-g_{\mu}^{\sigma_{\rho}^{x_{0}}}(0)\right) \xi_{\mu}=\sum_{j_{\mu} \in \mathbb{Z}}\left(g_{\mu}^{\theta}(x)-g_{\mu}^{\theta}(0)\right) \xi_{\mu} \text {. }
$$

This gives the first result of the Lemma. The second one is another direct application of the scaling result (100).

Case $\alpha=1$. The canonical basis of $\mathbb{R}^{d}$ is denoted by $\left(e_{1}, \ldots, e_{d}\right)$. We know from our construction that $\partial_{i} \Phi_{\mu}(x) \sim \delta\left(l_{i}, 1\right)$, when $x \rightarrow \mu=$ $(j, k, l)$, where $\delta$ denotes the Kronecker symbol, and at the same time

$$
\left|\partial_{i} \Phi_{\mu}(x)\right| \leq K \frac{1}{(1+|x-\mu|)^{d+\gamma}}
$$

Then, if $\rho \rightarrow 0^{+}$, using the proof that led to the uniform modulus result in the critical case $\alpha=1$, we get

$$
\begin{gathered}
\frac{1}{\sqrt{\log (1 / \rho)}} \sum_{\mu \in \Lambda} \frac{\Phi_{\mu}\left(x_{0}+\rho x\right)-\Phi_{\mu}\left(x_{0}\right)}{\rho} \xi_{\mu} \\
\sim \sum_{i=1}^{d} \frac{\left(x \mid e_{i}\right)}{\sqrt{\log (1 / \rho)}} \sum_{0 \leq j \leq \log (1 / \rho)} \xi_{\mu_{j}\left(x_{0}, i\right)},
\end{gathered}
$$

where $\mu_{j}(x, i)$ is defined by $\mu_{j}(x, i)=(j, k, l)$ if and only if $l=\delta(\cdot, i)$ and $x$ belong to a dyadic cube $q_{j, k}$. The end of the proof is now an application of the Central Limit Theorem.

As an immediate consequence we can now prove Theorem 1.4, 1.5.

\subsection{Local scalings for $X_{A}$.}

We first prove Theorems 1.4 and 1.5.

Lemma 5.2 gives ii) implies i) for both Theorem 1.4 and Theorem 1.5. As i) implies iii) is clear, we have only to prove iii) implies ii).

Let us consider the symbols

$$
\underline{\theta}(\xi):=\liminf _{\rho \rightarrow 0^{+}} \sigma_{\rho}^{x_{0}}(x, \xi), \quad \bar{\theta}(\xi):=\limsup _{\rho \rightarrow 0^{+}} \sigma_{\rho}^{x_{0}}(x, \xi)
$$


and recall that they satisfy (102) (see Lemma 5.1).

In the case $\alpha<1$, we deduce from the result (60) and the hypothesis of convergence

$$
\begin{aligned}
0 & <\int \frac{\sin ^{2}(u \xi)}{\bar{\theta}(\xi)} d \xi \\
& =\lim _{\rho \rightarrow 0^{+}} \mathbb{E}\left(\frac{X\left(x_{0}+\rho u\right)-X\left(x_{0}\right)^{2}}{\rho^{2 \alpha}}\right) \\
& =\int \frac{\sin ^{2}(u \xi)}{\underline{\theta}(\xi)} d \xi<\infty .
\end{aligned}
$$

This leads to the almost everywhere equality

$$
\underline{\theta}(\xi)=\bar{\theta}(\xi),
$$

and then to existence of the limit stated in ii).

In the case $\alpha=1$ the proof is the same, except that we use (63) instead of (60).

We now prove Proposition 1.3.

The fact that $\sigma=\sigma_{0} f$ satisfies HA $(m, \gamma)$ and that Proposition 1.1 can be applied is easy to check.

As we know that

$$
\lim _{\rho \rightarrow \infty} \frac{\sigma_{0}(\rho \xi)}{\rho^{d+2 \alpha}}=\theta_{x_{0}}(\xi)
$$

(see (38)) and also that the function $f$ is bounded we obtain the existence of

$$
\limsup _{\rho \rightarrow \infty} \frac{\sigma(\rho \xi)}{\rho^{d+2 \alpha}}
$$

Then, with the same arguments as in the proof of Lemma 5.2, case $\alpha<1$, we see that the process $X_{A}$ belongs to the weak L.A.S.S. class.

Moreover the process $X_{A}$ belongs to the L.A.S.S. class if and only if

$$
\lim _{\rho \rightarrow \infty} \frac{\sigma(\rho \xi)}{\rho^{d+2 \alpha}}
$$

exists and this, within our hypotheses, is equivalent to the existence of $\lim _{\rho \rightarrow \infty} f(\xi)$. 


\section{References.}

[1] Benassi, A., Théorème de traces stochastiques et fonctionnelles multiplicatives pour des champs gaussiens markoviens d'ordre p. Zeit. für Wahr. 59 (1982), 333-354.

[2] Benassi, A., Locally selfsimilar Gaussian processes. In Wavelets and Statistics. Ed. A. Antoniadis and G. Oppenheim. Lecture Notes in Statistics 103 (1995), 43-54. Springer-Verlag.

[3] Benassi, A., Jaffard, S., Roux, D., Analyse multiéchelle des champs gaussiens markoviens d'ordre $p$ indexés par $[0,1]$. Stochastic Processes and their Applications 47 (1993), 275-297.

[4] Benassi, A., Jaffard, S., Roux, D., Module de continuité des champs aléatoires gaussiens étudiés au moyen d'ondelettes appropriées. $C$. $R$. Acad. Sci. Paris 315 (1992), 441-446.

[5] Benassi, A., Oppenheim, G., Simulation et analyse multirésolution du mouvement Brownien fractionnaire d'indice $\alpha$. Preprint, 1994.

[6] Benfatto, G., Cassandro, M., Gallavotti, G., Nicolô , F., Olivieri, E., Presutti, E., Scacciatelli, E., Some probabilistic techniques in field theory. Comm. Math. Phys. 59 (1978), 143-166.

[7] Benfatto, G., Cassandro, M., Gallavotti, G., Nicolô, F., Olivieri, E., Presutti, E., Scacciatelli, E., Ultraviolet stability in euclidean scalar field theories. Comm. Math. Phys. 71 (1980), 95-130.

[8] Benfatto, G., Gallavotti, G., Nicolô, F., Elliptic equations and gaussian processes. J. Funct. Anal. 36 (1980), 343-400.

[9] Bourdaud, G., Réalisation des espaces de Besov homogènes. Arkiv. Math. 26 (1988), 41-54.

[10] Benjamini, I., Peres, Y., Tree indexed random walks on groups and first passage percolation. Probab. Theor. Relat. Fields. 98 (1994), 91-118.

[11] Ciesielski, Z., Kerkyacharian, G., Roynette, B., Quelques espaces fonctionnels associés ci des processus gaussiens. Studia Math. 107 (1993), 177-204.

[12] Coifman, R., Meyer, Y., Au dela des opérateurs pseudo-différentiels. Astérisque $\mathbf{5 7}$ (1978).

[13] Dobrushin, R. L., Gaussian and their subordinated self similar random fields. Ann. Probab. 7 (1979), 1-28.

[14] Donoho, D. L., Wavelet shrinkage and Wavelet-Vaguelette decomposition. Progress in wavelet analysis and applications. Ed. Y. Meyer and S. Roques. Edition Frontières, 1993.

[15] Dudley, R. M., Sample functions of the Gaussian processes. Ann. Probab. 1 (1973), 66-103. 
[16] Fernique, X., Régularité des trajectoires des fonctions aléatoires gaussiennes. Lecture Notes in Math. 480, Springer-Verlag, 1975.

[17] Guyon, X., Variations et identification de champs gaussiens markoviens sur $\mathbb{R}^{2}$. Actes du Colloque de Probabilités Numériques. C.I.R.M. Luminy, 1992.

[18] Houdré, C., Wavelets, Probability and Statistics: Some Bridges Wavelets. Mathematics and applications. Ed. J. J. Benedetto and M. Frazier. Studies in Advanced Mathematics, CRC Press (1994), 365-398.

[19] Inoué, K., Equivalence of measures for some class of gaussian fields. $J$. Multivariate Analysis 6 (1976), 295-308.

[20] Jaffard, S., Exposants de Hölder en des points donnés et coefficients d'ondelettes. C. R. Acad. Sci. Paris 308 (1989), 79-81.

[21] Jaffard, S., Propriétés des matrices "bien localisées" près de leur diagonale et quelques applications. Ann. Inst. H. Poincaré, Analyse non linéaire 7 (1990), 461-476.

[22] Jaffard, S., Meyer, Y., Bases d'ondelettes dans des ouverts de $\mathbb{R}^{n}$. J. Math. Pures et Appl. 68 (1989), 95-108.

[23] Kallianpur, G., Mandrekar, V., The Markov property for generalized gaussian random fields. Ann. Inst. Fourier 24 (1974), 143-167.

[24] Kerkacharian, G., Roynette, B., Une démonstration simple des théorèmes de Kolmogorov, Donsker et Itô-Nisio. C. R. Acad. Sci. Paris 312 (1991), 877-882.

[25] Kolmogorov, A. M., A refinement of previous hypotheses concerning the local structure of turbulence in viscous incompressible fluid at high Reynolds number. Journal of Fluid Mech. 177 (1961), 133-166.

[26] Kono, N., On the modulus of continuity of sample functions of gaussian processes. J. Math. Kyoto Univ. 10 (1970), 493-539.

[27] Ledoux, M., Talagrand, M., Probability in Banach spaces. Ergebnisse der Mathematik und ihrer Grenzgebiete 23. Springer-Verlag, 1991.

[28] Mandelbrot, B., Van Ness, J., Fractional brownian motion, fractional noises and applications. SIAM J. Rev. 10 (1968), 422-437.

[29] Marcus, M. M., Gaussian lacunary series and the Modulus of continuity for Gaussian Processes. Zeit. für Wahr. 22 (1972), 301-322.

[30] Meyer, Y., Ondelettes et opérateurs. Hermann, 1990.

[31] Molchan, G. M., On some points concerning Brownian motion in Lévy's sense. Theor. Probab. and Appl. 12, 682-690.

[32] Neveu, J., Processus aléatoires gaussiens. Séminaire d'été 1968, Presses de l'université de Montréal, 1968.

[33] Onural, L., Generating connected textured fractals patterns using Markov random fields. IEEE Trans. Comm. B 8 (1991), 103-127. 
[34] Sellan, F., Ph. D. Dissertation. To Appear. Université Paris Dauphine, 1997.

[35] Taqqu, M., Selfsimilar processes and related ultraviolet and infrared catastrophe Random fields. Coll. Math. Soc. Janos Bolyai 27 (1979), 1057-1096.

[36] Trèves, Introduction to pseudodifferential and Fourier integral operators. The University Series in Mathematics, Plenum Press, 1980.

Recibido: 7 de septiembre de 1.995

Revisado: 30 de julio de 1.996

Albert Benassi, Daniel Roux

Université Blaise Pascal

U.R.A. 1501 C.N.R.S., Les Cézeaux

63170 Aubiére, FRANCE

benassi@ucfma.univ-bpclermont.fr

roux@ucfma.univ-bpclermont.fr

and

Stéphane Jaffard

Département de Mathématiques Université Paris XII, Faculté des Sciences et Technologie

61 Av. du Président Wilson 94235 Cachan Cedex, FRANCE jaffard@umla.eus-cachan.fr 